\title{
A Novel Rough Set Model in Generalized Single Valued Neutrosophic Approximation Spaces and Its Application
}

\author{
Zhi-Lian Guo ${ }^{1}$, Yan-Ling Liu ${ }^{2}$ and Hai-Long Yang ${ }^{2, *}$ \\ 1 College of Economics, Northwest University of Political Science and Law, Xi'an 710063, China; \\ gzl_434@163.com \\ 2 College of Mathematics and Information Science, Shaanxi Normal University, Xi'an 710119, China; \\ 18829073422@163.com \\ * Correspondence: yanghailong@snnu.edu.cn; Tel.: +86-135-7210-6227
}

Received: 18 June 2017; Accepted: 11 July 2017; Published: 17 July 2017

\begin{abstract}
In this paper, we extend the rough set model on two different universes in intuitionistic fuzzy approximation spaces to a single-valued neutrosophic environment. Firstly, based on the $(\alpha, \beta, \gamma)$-cut relation $\widetilde{R}_{\{(\alpha, \beta, \gamma)\}}$, we propose a rough set model in generalized single-valued neutrosophic approximation spaces. Then, some properties of the new rough set model are discussed. Furthermore, we obtain two extended models of the new rough set model—the degree rough set model and the variable precision rough set model—and study some of their properties. Finally, we explore an example to illustrate the validity of the new rough set model.
\end{abstract}

Keywords: neutrosophic sets; single-valued neutrosophic sets; generalized single-valued neutrosophic approximation spaces; rough sets; single-valued neutrosophic relations

\section{Introduction}

Smarandache [1,2] introduced the concept of the neutrosophic set (NS), which consists of three membership functions (truth membership function, indeterminacy membership function and falsity membership function), where each function value is a real standard or nonstandard subset of the nonstandard unit interval $\left[0^{-}, 1^{+}\right]$. The neutrosophic set generalizes the concepts of the classical set, fuzzy set [3], interval-valued fuzzy set [4], intuitionistic fuzzy set [5] and interval-valued intuitionistic fuzzy set [6]. The neutrosophic set model is an important tool for dealing with real scientific and engineering applications because it can handle not only incomplete information, but also the inconsistent information and indeterminate information that exist commonly in real situations.

For easily applying NSs in the real world, Smarandache [1] and Wang et al. [7] proposed single-valued neutrosophic sets (SVNSs) by simplifying NSs. SVNSs can also be seen as an extension of intuitionistic fuzzy sets [5], in which three membership functions are unrelated and their function values belong to the unit closed interval. SVNSs has been a hot research issue. Ye [8,9] proposed decision making based on correlation coefficients and weighted correlation coefficients of SVNSs and illustrated the application of the proposed methods. Baušys et al. [10] applied SVNSs to multi-criteria decision making and proposed a new extension of the crisp complex proportional assessment (COPRAS) method named COPRAS-SVNS. Zavadskas et al. [11] applied SVNSs to the weighted aggregated sum product assessment (WASPAS) method, named WASPAS-SVNS, and used the new method to solve sustainable assessment of alternative sites for the construction of a waste incineration plant. Zavadskas et al. [12] also applied WASPAS-SVNS to the selection of a lead-zinc flotation circuit design. Zavadskas et al. [13] proposed a single-valued neutrosophic multi-attribute market value assessment method and applied this method to the sustainable market valuation of 
Croydon University Hospital. Li et al. [14] applied the Heronian mean to the neutrosophic set, proposed some Heronian mean operators and illustrated their application in multiple attribute group decision making. Baušys and Juodagalvienè [15] demonstrated garage location selection for a residential house. In [16], Ye proposed similarity measures between interval neutrosophic sets and applied them to multi-criteria decision making problems under the interval neutrosophic environment. Ye [17] proposed three vector similarity measures of simplified neutrosophic sets and applied them to a multi-criteria decision making problem with simplified neutrosophic information. Majumdar and Samanta [18] studied the distance, similarity and entropy of SVNSs from a theoretical aspect. Peng et al. [19] developed a new outranking approach for multi-criteria decision making problems in the context of a simplified neutrosophic environment. Liu and Wang [20] introduced an interval neutrosophic prioritized ordered weighted aggregation operator w.r.t. interval neutrosophic numbers and discussed its application in multiple attribute decision making. To deal with difficulties in steam turbine fault diagnosis, Zhang et al. [21] investigated a single-valued neutrosophic multi-granulation rough set over two universes. Şahin [22] proposed two kinds of interval neutrosophic cross-entropies based on the extension of fuzzy cross-entropy and single-valued neutrosophic cross-entropy and developed two multi-criteria decision making methods using the interval neutrosophic cross-entropy. Ye [23] proposed similarity measures between SVNSs based on the tangent function and a multi-period medical diagnosis method based on the similarity measure and the weighted aggregation of multi-period information to solve multi-period medical diagnosis problems with single-valued neutrosophic information. Yang et al. [24] proposed SVNRs and studied some kinds of kernels and closures of SVNRs. Ye [25] presented a simplified neutrosophic harmonic averaging projection measure and its multiple attribute decision making method with simplified neutrosophic information. Stanujkic et al. [26] proposed a new extension of the multi-objective optimization (MULTIMOORA) method adapted for usage with a neutrosophic set.

Rough set theory, initiated by Pawlak $[27,28]$, is a mathematical tool for the study of intelligent systems characterized by insufficient and incomplete information. The theory has been successfully applied to many fields, such as machine learning, knowledge acquisition, decision analysis, etc. To extend the application domain of rough set theory, more and more researchers have made some efforts toward the study of rough set models based on two different universes [29-39].

In recent years, many researchers have paid attention to combining neutrosophic sets with rough sets. Salama and Broumi [40] investigated the roughness of neutrosophic sets. Broumi and Smarandache put forward rough neutrosophic sets [41,42], as well as interval neutrosophic rough sets [43]. Yang et al. [44] proposed single-valued neutrosophic rough sets, which comprise a hybrid model of single-valued neutrosophic sets and rough sets. Along this line, this paper attempts to do some work regarding the fusion of single-valued neutrosophic sets and rough sets again. Concretely, we will extend the rough set model in [29] to a single-valued neutrosophic environment. Furthermore, we will apply the new model to a multi-attribute decision making problem.

The rest of this paper is organized as follows. In Section 2, we recall some basic notions related to Pawlak rough sets, SVNSs and single-valued neutrosophic rough sets. In Section 3, we propose a rough set model in generalized single-valued neutrosophic approximation spaces. Section 4 gives two extended models and studies some related properties. Section 5 explores an example to illustrate the new rough set model's application in multi-attribute decision making. The last section summarizes the conclusions.

\section{Preliminaries}

In this section, we recall some basic notions of Pawlak rough sets, SVNSs and single-valued neutrosophic rough sets. 


\subsection{Pawlak Rough Sets}

Definition 1. ([27,28]) Let $U$ be a nonempty finite universe and $R$ be an equivalence relation in $U .(U, R)$ is called a Pawlak approximation space. $\forall X \subseteq U$, the lower and upper approximations of $X$, denoted by $\underline{R}(X)$ and $\bar{R}(X)$, are defined as follows, respectively:

$$
\begin{aligned}
& \underline{R}(X)=\left\{x \in U \mid[x]_{R} \subseteq X\right\}, \\
& \bar{R}(X)=\left\{x \in U \mid[x]_{R} \cap X \neq \varnothing\right\},
\end{aligned}
$$

where $[x]_{R}=\{y \in U \mid(x, y) \in R\} . \underline{R}$ and $\bar{R}$ are called the lower and upper approximation operators, respectively. The pair $(\underline{R}(X), \bar{R}(X))$ is called a Pawlak rough set.

Furthermore, the positive region, boundary region and negative region of the subset $X$ are defined by

$$
\operatorname{pos}(X)=\underline{R}(X), \quad \operatorname{neg}(X)=U-\bar{R}(X), \quad \operatorname{bn}(X)=\bar{R}(X)-\underline{R}(X) .
$$

\subsection{Single-Valued Neutrosophic Sets and Single-Valued Neutrosophic Rough Sets}

Definition 2. ([7]) Let $U$ be a space of points (objects), with a generic element in $U$ denoted by $x$. A SVNS $\widetilde{A}$ in $U$ is characterized by three membership functions, a truth membership function $T_{\widetilde{A}}$, an indeterminacy membership function $I_{\widetilde{A}}$ and a falsity membership function $F_{\widetilde{A}}$, where $\forall x \in U, T_{\widetilde{A}}(x), I_{\widetilde{A}}(x), F_{\widetilde{A}}(x) \in[0,1]$.

The SVNS $\widetilde{A}$ can be denoted by $\widetilde{A}=\left\{\left\langle x, T_{\widetilde{A}}(x), I_{\widetilde{A}}(x), F_{\widetilde{A}}(x)\right\rangle \mid x \in U\right\}$ or $\widetilde{A}=\left(T_{\widetilde{A}}, I_{\widetilde{A}}, F_{\widetilde{A}}\right)$. $\forall x \in U, \widetilde{A}(x)=\left(T_{\widetilde{A}}(x), I_{\widetilde{A}}(x), F_{\widetilde{A}}(x)\right)$, and $\left(T_{\widetilde{A}}(x), I_{\widetilde{A}}(x), F_{\widetilde{A}}(x)\right)$ is called a single-valued neutrosophic number.

Definition 3. ([44]) An SVNS $\widetilde{R}$ in $U \times U$ is called a single-valued neutrosophic relation (SVNR) in $U$, denoted by $\widetilde{R}=\left\{\left\langle(x, y), T_{\widetilde{R}}(x, y), I_{\widetilde{R}}(x, y), F_{\widetilde{R}}(x, y)\right\rangle \mid(x, y) \in U \times U\right\}$, where $T_{\widetilde{R}}: U \times U \longrightarrow[0,1]$, $I_{\widetilde{R}}: U \times U \longrightarrow[0,1]$ and $F_{\widetilde{R}}: U \times U \longrightarrow[0,1]$ denote the truth membership function, indeterminacy membership function and falsity membership function of $\widetilde{R}$, respectively.

Definition 4. ([45]) Let $\widetilde{R}, \widetilde{S}$ be two SVNRs in $U$. If $\forall x, y \in U, T_{\widetilde{R}}(x, y) \leq T_{\widetilde{S}}(x, y), I_{\widetilde{R}}(x, y) \geq I_{\widetilde{S}}(x, y)$ and $F_{\widetilde{R}}(x, y) \geq F_{\widetilde{S}}(x, y)$, then we say $\widetilde{R}$ is contained in $\widetilde{S}$, denoted by $\widetilde{R} \widetilde{\subseteq} \widetilde{S}$. In other words, we say $\widetilde{S}$ contains $\widetilde{R}$, denoted by $\widetilde{S} \supseteq \widetilde{R}$.

Definition 5. ([24]) Let $\widetilde{R}$ be an SVNR in $U$. If $\forall x \in U, T_{\widetilde{R}}(x, x)=1$ and $I_{\widetilde{R}}(x, x)=F_{\widetilde{R}}(x, x)=0$, then $\widetilde{R}$ is called a reflexive SVNR. If $\forall x, y \in U, T_{\widetilde{R}}(x, y)=T_{\widetilde{R}}(y, x)$, $I_{\widetilde{R}}(x, y)=I_{\widetilde{R}}(y, x)$ and $F_{\widetilde{R}}(x, y)=F_{\widetilde{R}}(y, x)$, then $\widetilde{R}$ is called a symmetric SVNR. If $\forall x \in U, \vee_{y \in U} T_{\widetilde{R}}(x, y)=1$ and $\bigwedge_{y \in U} I_{\widetilde{R}}(x, y)=\Lambda_{y \in U} F_{\widetilde{R}}(x, y)=0$, then $\widetilde{R}$ is called a serial SVNR. If $\forall x, y, z \in U, \vee_{y \in U}\left(T_{\widetilde{R}}(x, y) \wedge T_{\widetilde{R}}(y, z)\right) \leq T_{\widetilde{R}}(x, z), \wedge_{y \in U}\left(I_{\widetilde{R}}(x, y) \vee I_{\widetilde{R}}(y, z)\right) \geq I_{\widetilde{R}}(x, z)$ and $\Lambda_{y \in U}\left(F_{\widetilde{R}}(x, y) \vee F_{\widetilde{R}}(y, z)\right) \geq F_{\widetilde{R}}(x, z)$, then $\widetilde{R}$ is called a transitive $S V N R$, where " $\vee$ " and " $\wedge$ " denote maximum and minimum, respectively.

Definition 6. ([24]) Let $\widetilde{R}$ be an SVNR in $U$; the tuple $(U, \widetilde{R})$ is called a single-valued neutrosophic approximation space. $\forall \widetilde{A} \in S V N S(U)$, the lower and upper approximations of $\widetilde{A}$ w.r.t. $(U, \widetilde{R})$, denoted by $\underline{\widetilde{R}}(\widetilde{A})$ and $\overline{\widetilde{R}}(\widetilde{A})$, are two SVNSs whose membership functions are defined as: $\forall x \in U$,

$T_{\widetilde{R}(\widetilde{A})}(x)=\bigwedge_{y \in U}\left(F_{\widetilde{R}}(x, y) \vee T_{\widetilde{A}}(y)\right)$,

$I_{\widetilde{R}(\widetilde{A})}(x)=\bigvee_{y \in U}\left(\left(1-I_{\widetilde{R}}(x, y)\right) \wedge I_{\widetilde{A}}(y)\right)$,

$F_{\widetilde{R}(\widetilde{A})}(x)=\bigvee_{y \in U}\left(T_{\widetilde{R}}(x, y) \wedge F_{\widetilde{A}}(y)\right)$;

$T_{\widetilde{R}(\widetilde{A})}(x)=\bigvee_{y \in U}\left(T_{\widetilde{R}}(x, y) \wedge T_{\widetilde{A}}(y)\right)$,

$I_{\widetilde{R}(\widetilde{A})}(x)=\bigwedge_{y \in U}\left(I_{\widetilde{R}}(x, y) \vee I_{\widetilde{A}}(y)\right)$,

$F_{\widetilde{R}(\widetilde{A})}(x)=\bigwedge_{y \in U}\left(F_{\widetilde{R}}(x, y) \vee F_{\widetilde{A}}(y)\right)$. 
The pair $(\widetilde{R}(\widetilde{A}), \overline{\widetilde{R}}(\widetilde{A}))$ is called the single-valued neutrosophic rough set of $\widetilde{A}$ w.r.t. $\quad(U, \widetilde{R})$. $\underline{\widetilde{R}}$ and $\overline{\widetilde{R}}$ are referred to as the single-valued neutrosophic lower and upper approximation operators, respectively.

\section{Rough Set Model in Generalized Single-Valued Neutrosophic Approximation Spaces}

Guo et al. [29] studied the rough set model on two different universes in intuitionistic fuzzy approximation space. In this section, we will extend the rough set model in [29] to a single-valued neutrosophic environment.

Yang et al. [24] proposed the notions of single-valued neutrosophic relations from $U$ to $V$ and generalized single-valued neutrosophic approximation spaces as follows.

Definition 7. ([24]) Let $U$ and $V$ be two nonempty finite universes. The relation $\widetilde{R}$ in $U \times V$ is called a single-valued neutrosophic relation from $U$ to $V$, denoted by $\widetilde{R}=\left\{\left\langle(x, y), T_{\widetilde{R}}(x, y), I_{\widetilde{R}}(x, y), F_{\widetilde{R}}(x, y)\right\rangle \mid(x, y) \in U \times V\right\}, \quad$ where $\quad T_{\widetilde{R}}: U \times V \longrightarrow[0,1]$, $I_{\widetilde{R}}: U \times V \longrightarrow[0,1]$ and $F_{\widetilde{R}}: U \times V \longrightarrow[0,1]$ denote the truth membership function, indeterminacy membership function and falsity membership function of $\widetilde{R}$, respectively.

The triple $(U, V, \widetilde{R})$ is called a generalized single-valued neutrosophic approximation space on two different universes.

Remark 1. If $U=V$, then we call $\widetilde{R}$ a single-valued neutrosophic relation in $U$.

Definition 8. Let $\widetilde{R}$ be an $S V N R$ from $U$ to $V$. If $\forall x \in U, y \in V, T_{\widetilde{R}}(x, y)=T_{\widetilde{R}}(y, x), I_{\widetilde{R}}(x, y)=I_{\widetilde{R}}(y, x)$ and $F_{\widetilde{R}}(x, y)=F_{\widetilde{R}}(y, x)$, then $\widetilde{R}$ is called a symmetric SVNR. If $\forall x \in U, \bigvee_{y \in V} T_{\widetilde{R}}(x, y)=1$ and $\bigwedge_{y \in V} I_{\widetilde{R}}(x, y)=\bigwedge_{y \in V} F_{\widetilde{R}}(x, y)=0$, then $\widetilde{R}$ is called a serial SVNR.

The union, intersection and containmentof two SVNRs from $U$ to $V$ are defined as follows, respectively.

Definition 9. Let $R, S$ be two SVNRs from $U$ to $V$.

(1) The union $R \widetilde{\cup} S$ of $R$ and $S$ is defined by $R \widetilde{\cup S}=\left\{\left\langle(x, y), \max \left\{T_{R}(x, y), T_{S}(x, y)\right\}\right.\right.$, $\left.\left.\min \left\{I_{R}(x, y), I_{S}(x, y)\right\}, \min \left\{F_{R}(x, y), F_{S}(x, y)\right\}\right\rangle \mid(x, y) \in U \times V\right\}$.

(2) The intersection $R \widetilde{\cap} S$ of $R$ and $S$ is defined by $R \widetilde{\cap} S=\left\{\left\langle(x, y), \min \left\{T_{R}(x, y), T_{S}(x, y)\right\}\right.\right.$, $\left.\left.\max \left\{I_{R}(x, y), I_{S}(x, y)\right\}, \max \left\{F_{R}(x, y), F_{S}(x, y)\right\}\right\rangle \mid(x, y) \in U \times V\right\}$.

(3) If $\forall(x, y) \in U \times V, T_{R}(x, y) \leq T_{S}(x, y), I_{R}(x, y) \geq I_{S}(x, y)$ and $F_{R}(x, y) \geq F_{S}(x, y)$, then we say $R$ is contained in $S$, denoted by $R \widetilde{\subseteq} S$.

Next, we give the notion of $(\alpha, \beta, \gamma)$-cut relation $\widetilde{R}_{\{(\alpha, \beta, \gamma)\}}$ of a single-valued neutrosophic relation $\widetilde{R}$ from $U$ to $V$.

Definition 10. Let $U, V$ be two nonempty finite universes and $\widetilde{R}$ be a single-valued neutrosophic relation from $U$ to $V$. For any $\alpha, \beta, \gamma \in(0,1]$, we define the $(\alpha, \beta, \gamma)$-cut relation $\widetilde{R}_{\{(\alpha, \beta, \gamma)\}}$ of $\widetilde{R}$ as follows:

$$
\widetilde{R}_{\{(\alpha, \beta, \gamma)\}}=\left\{(x, y) \in U \times V \mid T_{\widetilde{R}}(x, y) \geq \alpha, I_{\widetilde{R}}(x, y) \leq \beta, F_{\widetilde{R}}(x, y) \leq \gamma\right\} .
$$

According to Definition 10, if $(x, y) \in \widetilde{R}_{\{(\alpha, \beta, \gamma)\}}$, it indicates that the truth membership degree of the relationships of $x$ and $y$ w.r.t. SVNR $\widetilde{R}$ is not less than $\alpha$, and the indeterminacy membership degree and falsity membership degree of the relationships of $x$ and $y$ w.r.t. SVNR $\widetilde{R}$ are not more than $\beta$ and $\gamma$, respectively. 
Definition 11. Let $(U, V, \widetilde{R})$ be a generalized single-valued neutrosophic approximation space. $\widetilde{R}_{\{(\alpha, \beta, \gamma)\}}$ is the $(\alpha, \beta, \gamma)$-cut relation defined in Definition 8. For any $x \in U, y \in V$, we define

$$
\begin{aligned}
& \widetilde{R}_{\{(\alpha, \beta, \gamma)\}}(x)=\left\{y \in V \mid T_{\widetilde{R}}(x, y) \geq \alpha, I_{\widetilde{R}}(x, y) \leq \beta, F_{\widetilde{R}}(x, y) \leq \gamma\right\}, \\
& \widetilde{R}_{\{(\alpha, \beta, \gamma)\}}^{-1}(y)=\left\{x \in U \mid T_{\widetilde{R}}(x, y) \geq \alpha, I_{\widetilde{R}}(x, y) \leq \beta, F_{\widetilde{R}}(x, y) \leq \gamma\right\} .
\end{aligned}
$$

The following Definition 12 gives a rough set model on two universes based on the $(\alpha, \beta, \gamma)$-cut relation $\widetilde{R}_{\{(\alpha, \beta, \gamma)\}}$ induced by a single-valued neutrosophic relation $\widetilde{R}$ from $U$ to $V$.

Definition 12. Let $(U, V, \widetilde{R})$ be a generalized single-valued neutrosophic approximation space. Suppose $\widetilde{R}_{\{(\alpha, \beta, \gamma)\}}$ is the $(\alpha, \beta, \gamma)$-cut relation given in Definition 10 from $U$ to $V$. For any set $Y \subseteq V$, the lower approximation and upper approximation of $Y$ on two universes w.r.t. $(U, V, \widetilde{R})$ and $(\alpha, \beta, \gamma)$ are defined by

$$
\begin{aligned}
& \widetilde{R}_{\{(\alpha, \beta, \gamma)\}}(Y)=\left\{x \in U \mid \widetilde{R}_{\{(\alpha, \beta, \gamma)\}}(x) \subseteq Y \text { and } \widetilde{R}_{\{(\alpha, \beta, \gamma)\}}(x) \neq \varnothing\right\}, \\
& \overline{\widetilde{R}}_{\{(\alpha, \beta, \gamma)\}}(Y)=\left\{x \in U \mid \widetilde{R}_{\{(\alpha, \beta, \gamma)\}}(x) \cap Y \neq \varnothing \text { or } \widetilde{R}_{\{(\alpha, \beta, \gamma)\}}(x)=\varnothing\right\}
\end{aligned}
$$

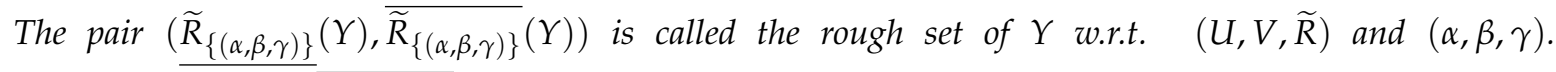
If $\widetilde{R}_{\{(\alpha, \beta, \gamma)\}}(Y)=\widetilde{R}_{\{(\alpha, \beta, \gamma)\}}(Y)$, then $Y$ is called the definable set w.r.t. $(U, V, \widetilde{R})$ and $(\alpha, \beta, \gamma)$.

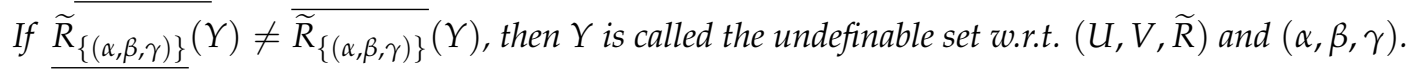

Next, we define the positive region $\operatorname{pos}_{\widetilde{R}_{\{(\alpha, \beta, \gamma)\}}}(Y)$, negative region neg $\widetilde{R}_{\{(\alpha, \beta, \gamma)\}}(Y)$ and boundary region $\operatorname{bn}_{\widetilde{R}_{\{(\alpha, \beta, \gamma)\}}}(Y)$ of $Y$, respectively:

$$
\begin{aligned}
& \operatorname{pos}_{\widetilde{R}_{\{(\alpha, \beta, \gamma)\}}}(Y)=\underline{\widetilde{R}_{\{(\alpha, \beta, \gamma)\}}(Y),} \\
& n e g_{\widetilde{R}_{\{(\alpha, \beta, \gamma)\}}}(Y)=\overline{U-\widetilde{R}_{\{(\alpha, \beta, \gamma)\}}}(Y), \\
& b n_{\widetilde{R}_{\{(\alpha, \beta, \gamma)\}}}(Y)=\overline{\widetilde{R}}_{\{(\alpha, \beta, \gamma)\}}(Y)-\underline{\widetilde{R}_{\{(\alpha, \beta, \gamma)\}}}(Y) \text {. }
\end{aligned}
$$

Remark 2. If $\widetilde{R}$ is a series single-valued neutrosophic relation from $U$ to $V$, i.e., $\forall x \in U, \bigvee_{y \in U} T_{\widetilde{R}}(x, y)=1$ and $\bigwedge_{y \in U} I_{\widetilde{R}}(x, y)=\bigwedge_{y \in U} F_{\widetilde{R}}(x, y)=0$, then there exists $y \in V$ such that $T_{\widetilde{R}}(x, y)=1$ and $I_{\widetilde{R}}(x, y)=0, F_{\widetilde{R}}(x, y)=0$ for all $x \in U$ since $V$ is finite. Therefore, for any $\alpha, \beta, \gamma \in(0,1]$, we have $\widetilde{R}_{\{(\alpha, \beta, \gamma)\}}(x) \neq \varnothing$. Therefore, we have

$$
\begin{aligned}
\widetilde{\widetilde{R}}_{\{(\alpha, \beta, \gamma)\}}(Y) & =\left\{x \in U \mid \widetilde{R}_{\{(\alpha, \beta, \gamma)\}}(x) \subseteq Y \text { and } \widetilde{R}_{\{(\alpha, \beta, \gamma)\}}(x) \neq \varnothing\right\}, \\
& =\left\{x \in U \mid \widetilde{R}_{\{(\alpha, \beta, \gamma)\}}(x) \subseteq Y\right\}, \\
\widetilde{\widetilde{R}}_{\{(\alpha, \beta, \gamma)\}}(Y) & =\left\{x \in U \mid \widetilde{R}_{\{(\alpha, \beta, \gamma)\}}(x) \cap Y \neq \varnothing \text { or } \widetilde{R}_{\{(\alpha, \beta, \gamma)\}}(x)=\varnothing\right\} . \\
& =\left\{x \in U \mid \widetilde{R}_{\{(\alpha, \beta, \gamma)\}}(x) \cap Y \neq \varnothing\right\} .
\end{aligned}
$$

In the following, we discuss some properties of the lower approximation and the upper approximation given in Definition 12.

Theorem 1. Let $(U, V, \widetilde{R})$ be a generalized single-valued neutrosophic approximation space. Suppose $\widetilde{R}_{\{(\alpha, \beta, \gamma)\}}$ is the $(\alpha, \beta, \gamma)$-cut relation given in Definition 10 from $U$ to $V$. For any $Y, Y_{1}, Y_{2} \subseteq V$, the following properties hold:

(1) $\quad \widetilde{R}_{\{(\alpha, \beta, \gamma)\}}(Y) \subseteq \widetilde{\widetilde{R}}_{\{(\alpha, \beta, \gamma)\}}(Y) ;$

(2) $\quad \widetilde{R}_{\{(\alpha, \beta, \gamma)\}}(\varnothing)=\varnothing, \widetilde{R}_{\{(\alpha, \beta, \gamma)\}}(V)=U$;

(3) $\quad \underline{\widetilde{R}_{\{(\alpha, \beta, \gamma)\}}}\left(Y_{1} \cap Y_{2}\right)=\underline{\widetilde{R}_{\{(\alpha, \beta, \gamma)\}}}\left(Y_{1}\right) \cap \underline{\widetilde{R}_{\{(\alpha, \beta, \gamma)\}}}\left(Y_{2}\right)$, 
$\begin{array}{ll} & \overline{\widetilde{R}_{\{(\alpha, \beta, \gamma)\}}}\left(Y_{1} \cup Y_{2}\right)=\overline{\widetilde{R}_{\{(\alpha, \beta, \gamma)\}}}\left(Y_{1}\right) \cup \overline{\widetilde{R}_{\{(\alpha, \beta, \gamma)\}}}\left(Y_{2}\right) ; \\ \text { (4) } \quad \widetilde{R}_{\{(\alpha, \beta, \gamma)\}}\left(Y_{1} \cup Y_{2}\right) \supseteq \widetilde{R}_{\{(\alpha, \beta, \gamma)\}}\left(Y_{1}\right) \cup \widetilde{R}_{\{(\alpha, \beta, \gamma)\}}\left(Y_{2}\right),\end{array}$

$$
\begin{aligned}
& \widetilde{R}_{\{(\alpha, \beta, \gamma)\}}\left(Y_{1} \cup Y_{2}\right)=\widetilde{R}_{\{(\alpha, \beta, \gamma)\}}\left(Y_{1}\right) \cup \widetilde{R}_{\{(\alpha, \beta, \gamma)\}}\left(Y_{2}\right) ; \\
& \underline{\overline{\widetilde{R}_{\{(\alpha, \beta, \gamma)\}}}}\left(Y_{1} \cup Y_{2}\right) \supseteq \overline{\widetilde{R}_{\{(\alpha, \beta, \gamma)\}}}\left(Y_{1}\right) \cup \overline{\widetilde{R}_{\{(\alpha, \beta, \gamma)\}}}\left(Y_{2}\right), \\
& \overline{\widetilde{R}_{\{(\alpha, \beta, \gamma)\}}}\left(Y_{1} \cap Y_{2}\right) \subseteq \overline{\widetilde{R}_{\{(\alpha, \beta, \gamma)\}}}\left(Y_{1}\right) \cap \overline{\widetilde{R}_{\{(\alpha, \beta, \gamma)\}}}\left(Y_{2}\right) ;
\end{aligned}
$$

(5) If $Y_{1} \subseteq Y_{2}$, then $\underline{\widetilde{R}_{\{(\alpha, \beta, \gamma)\}}}\left(Y_{1}\right) \subseteq \underline{\widetilde{R}_{\{(\alpha, \beta, \gamma)\}}}\left(Y_{2}\right)$ and $\overline{\widetilde{R}_{\{(\alpha, \beta, \gamma)\}}}\left(Y_{1}\right) \subseteq \overline{\widetilde{R}_{\{(\alpha, \beta, \gamma)\}}}\left(Y_{2}\right)$;

(6) $\quad \widetilde{\widetilde{R}}_{\{(\alpha, \beta, \gamma)\}}(Y)=\overline{\sim \widetilde{\widetilde{R}}_{\{(\alpha, \beta, \gamma)\}}}(\sim Y), \overline{\widetilde{R}_{\{(\alpha, \beta, \gamma)\}}}(Y)=\sim \underline{\underline{\widetilde{R}_{\{(\alpha, \beta, \gamma)\}}}}(\sim Y)$.

Proof. We only prove (3) and (6).

$$
\begin{aligned}
& \text { (3) } \widetilde{R}_{\{(\alpha, \beta, \gamma)\}}\left(Y_{1} \cap Y_{2}\right) \\
& =\left\{x \in U \mid \widetilde{R}_{\{(\alpha, \beta, \gamma)\}}(x) \subseteq Y_{1} \cap Y_{2} \text { and } \widetilde{R}_{\{(\alpha, \beta, \gamma)\}}(x) \neq \varnothing\right\} \\
& =\left\{x \in U \mid \widetilde{R}_{\{(\alpha, \beta, \gamma)\}}(x) \subseteq Y_{1} \text { and } \widetilde{R}_{\{(\alpha, \beta, \gamma)\}}(x) \subseteq Y_{2} \text { and } \widetilde{R}_{\{(\alpha, \beta, \gamma)\}}(x) \neq \varnothing\right\} \\
& =\left\{x \in U \mid \widetilde{R}_{\{(\alpha, \beta, \gamma)\}}(x) \subseteq Y_{1} \text { and } \widetilde{R}_{\{(\alpha, \beta, \gamma)\}}(x) \neq \varnothing\right\} \cap\left\{x \in U \mid \widetilde{R}_{\{(\alpha, \beta, \gamma)\}}(x) \subseteq Y_{2}\right. \text { and } \\
& \left.\widetilde{R}_{\{(\alpha, \beta, \gamma)\}}(x) \neq \varnothing\right\} \\
& =\underline{\widetilde{R}_{\{(\alpha, \beta, \gamma)\}}}\left(Y_{1}\right) \cap \underline{\widetilde{R}_{\{(\alpha, \beta, \gamma)\}}}\left(Y_{2}\right) ; \\
& \overline{\widetilde{R}_{\{(\alpha, \beta, \gamma)\}}}\left(Y_{1} \cup Y_{2}\right) \\
& =\left\{x \in U \mid \widetilde{R}_{\{(\alpha, \beta, \gamma)\}}(x) \cap\left(Y_{1} \cup Y_{2}\right) \neq \varnothing \text { or } \widetilde{R}_{\{(\alpha, \beta, \gamma)\}}(x)=\varnothing\right\} \\
& =\left\{x \in U \mid\left(\widetilde{R}_{\{(\alpha, \beta, \gamma)\}}(x) \cap Y_{1}\right) \cup\left(\widetilde{R}_{\{(\alpha, \beta, \gamma)\}}(x) \cap Y_{2}\right) \neq \varnothing \text { or } \widetilde{R}_{\{(\alpha, \beta, \gamma)\}}(x)=\varnothing\right\} \\
& =\left\{x \in U \mid \widetilde{R}_{\{(\alpha, \beta, \gamma)\}}(x) \cap Y_{1} \neq \varnothing \text { or } \widetilde{R}_{\{(\alpha, \beta, \gamma)\}}(x)=\varnothing\right\} \cup\left\{x \in U \mid \widetilde{R}_{\{(\alpha, \beta, \gamma)\}}(x) \cap Y_{2} \neq \varnothing\right. \\
& \text { or } \left.\widetilde{R}_{\{(\alpha, \beta, \gamma)\}}(x)=\varnothing\right\}
\end{aligned}
$$

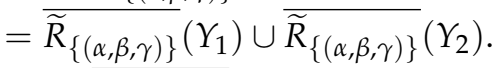

$$
\begin{aligned}
& \text { (6) } \sim \widetilde{R}_{\{(\alpha, \beta, \gamma)\}}(\sim Y) \\
& =\sim\left\{x \in U \mid \widetilde{R}_{\{(\alpha, \beta, \gamma)\}}(x) \cap(\sim Y) \neq \varnothing \text { or } \widetilde{R}_{\{(\alpha, \beta, \gamma)\}}(x)=\varnothing\right\} \\
& =\left\{x \in U \mid \widetilde{R}_{\{(\alpha, \beta, \gamma)\}}(x) \cap(\sim Y)=\varnothing \text { and } \widetilde{R}_{\{(\alpha, \beta, \gamma)\}}(x) \neq \varnothing\right\} \\
& =\left\{x \in U \mid \widetilde{R}_{\{(\alpha, \beta, \gamma)\}}(x) \subseteq Y \text { and } \widetilde{R}_{\{(\alpha, \beta, \gamma)\}}(x) \neq \varnothing\right\} \\
& =\widetilde{R}_{\{(\alpha, \beta, \gamma)\}}(Y) \text {; } \\
& \sim \widetilde{\widetilde{R}}_{\{(\alpha, \beta, \gamma)\}}(\sim Y) \\
& =\widetilde{\sim}\left\{x \in U \mid \widetilde{R}_{\{(\alpha, \beta, \gamma)\}}(x) \subseteq(\sim Y) \text { and } \widetilde{R}_{\{(\alpha, \beta, \gamma)\}}(x) \neq \varnothing\right\} \\
& =\sim\left\{x \in U \mid \widetilde{R}_{\{(\alpha, \beta, \gamma)\}}(x) \cap Y=\varnothing \text { and } x \in U \mid \widetilde{R}_{\{(\alpha, \beta, \gamma)\}}(x) \neq \varnothing\right\} \\
& =\left\{x \in U \mid \widetilde{R}_{\{(\alpha, \beta, \gamma)\}}(x) \cap Y \neq \varnothing \text { or } \widetilde{R}_{\{(\alpha, \beta, \gamma)\}}(x)=\varnothing\right\} \\
& =\overline{\widetilde{R}_{\{(\alpha, \beta, \gamma)}}(Y) \text {. }
\end{aligned}
$$

Remark 3. In general,

(1) $\quad \widetilde{\widetilde{R}}_{\{(\alpha, \beta, \gamma)\}}(\varnothing) \neq \varnothing, \underline{\widetilde{R}_{\{(\alpha, \beta, \gamma)\}}}(V) \neq U$;

(2) $\begin{aligned} & \widetilde{R}_{\{(\alpha, \beta, \gamma)\}} \\ & \left.\quad Y_{1} \cup Y_{2}\right) \neq \underline{\widetilde{R}_{\{(\alpha, \beta, \gamma)\}}}\left(Y_{1}\right) \cup \underline{\widetilde{R}_{\{(\alpha, \beta, \gamma)\}}}\left(Y_{2}\right) \text { and } \overline{\widetilde{R}_{\{(\alpha, \beta, \gamma)\}}}\left(Y_{1} \cap Y_{2}\right) \neq \overline{\widetilde{R}_{\{(\alpha, \beta, \gamma)\}}}\left(Y_{1}\right) \cap\end{aligned}$ as shown in the following example.

Example 1. Let $U=\left\{x_{1}, x_{2}, x_{3}\right\}, V=\left\{y_{1}, y_{2}, y_{3}, y_{4}\right\} . Y_{1}=\left\{y_{1}\right\}$ and $Y_{2}=\left\{y_{3}\right\}$. The single-valued neutrosophic relation $\widetilde{R}$ from $U$ to $V$ is given in Table 1 . 
Table 1. The single-valued neutrosophic relation $\widetilde{R}$ from $U$ to $V$.

\begin{tabular}{ccccc}
\hline$\widetilde{\boldsymbol{R}}$ & $y_{1}$ & $y_{\mathbf{2}}$ & $y_{3}$ & $y_{4}$ \\
\hline$x_{1}$ & $(0.7,0,1,0.2)$ & $(0.8,0.3,0.2)$ & $(0.7,0.2,0.1)$ & $(1,0,0)$ \\
$x_{2}$ & $(1,0.3,0.1)$ & $(0,0,1)$ & $(0.7,0.3,0.2)$ & $(0,0,1)$ \\
$x_{3}$ & $(0.2,0.1,0.8)$ & $(0.1,0.2,0.7)$ & $(0.8,0.2,0.1)$ & $(0,0.3,1)$ \\
\hline
\end{tabular}

(1) Take $\alpha=0.9, \beta=0.3$ and $\gamma=0.2$; we have

$\widetilde{R}_{\{(0.9,0.3,0.2)\}}\left(x_{1}\right)=\left\{y_{4}\right\}, \widetilde{R}_{\{(0.9,0.3,0.2)\}}\left(x_{2}\right)=\left\{y_{1}\right\}, \widetilde{R}_{\{(0.9,0.3,0.2)\}}\left(x_{3}\right)=\left\{y_{3}\right\}$.

By Definition 12, we have $\widetilde{R}_{\{(\alpha, \beta, \gamma)\}}(\varnothing)=\left\{x_{3}\right\} \neq \varnothing$ and $\underline{\widetilde{R}_{\{(\alpha, \beta, \gamma)\}}}(V)=\left\{x_{1}, x_{2}\right\} \neq U$.

(2) Take $\alpha=0.5, \beta=0.3$ and $\gamma=0.2$; we have

$\widetilde{R}_{\{(0.5,0.3,0.2)\}}\left(x_{1}\right)=\left\{y_{1}, y_{2}, y_{3}, y_{4}\right\}, \widetilde{R}_{\{(0.5,0.3,0.2)\}}\left(x_{2}\right)=\left\{y_{1}, y_{3}\right\}, \widetilde{R}_{\{(0.5,0.3,0.2)\}}\left(x_{3}\right)=\varnothing$.

By Definition 12, we have

$$
\begin{aligned}
& \underline{l \widetilde{R}_{\{(\alpha, \beta, \gamma)\}}}\left(Y_{1}\right)=\varnothing, \underline{\widetilde{R}_{\{(\alpha, \beta, \gamma)\}}}\left(Y_{2}\right)=\left\{x_{3}\right\}, \underline{\widetilde{R}_{\{(\alpha, \beta, \gamma)\}}}\left(Y_{1} \cup Y_{2}\right)=\left\{x_{2}, x_{3}\right\} . \\
& \overline{\widetilde{R}_{\{(\alpha, \beta, \gamma)\}}}\left(Y_{1}\right)=\left\{x_{1}, x_{2}\right\}, \widetilde{R}_{\{(\alpha, \beta, \gamma)\}}\left(Y_{2}\right)=\left\{x_{1}, x_{2}, x_{3}\right\}, \widetilde{R}_{\{(\alpha, \beta, \gamma)\}}\left(Y_{1} \cap Y_{2}\right)=\varnothing \text {. }
\end{aligned}
$$

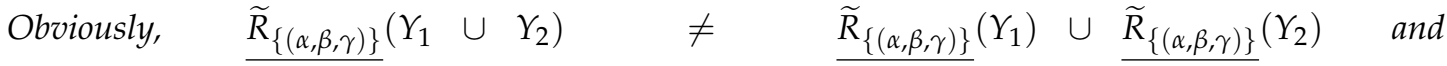
$\overline{\widetilde{R}_{\{(\alpha, \beta, \gamma)\}}}\left(Y_{1} \cap Y_{2}\right) \neq \widetilde{\widetilde{R}}_{\{(\alpha, \beta, \gamma)\}}\left(Y_{1}\right) \cap \widetilde{\widetilde{R}}_{\{(\alpha, \beta, \gamma)\}}\left(Y_{2}\right)$.

Theorem 2. Let $(U, V, \widetilde{R})$ be a generalized single-valued neutrosophic approximation space. $\widetilde{R}_{\left\{\left(\alpha_{1}, \beta_{1}, \gamma_{1}\right)\right\}}$ and $\widetilde{R}_{\left\{\left(\alpha_{2}, \beta_{2}, \gamma_{2}\right)\right\}}$ are two relations defined in Definition 10. If $\widetilde{R}$ is a series, $\alpha_{1} \leq \alpha_{2}, \beta_{1} \geq \beta_{2}$ and $\gamma_{1} \geq \gamma_{2}$, then

(1) $\underline{\widetilde{R}}_{\left\{\left(\alpha_{1}, \beta_{1}, \gamma_{1}\right)\right\}}(Y) \subseteq \underline{\widetilde{R}_{\left\{\left(\alpha_{2}, \beta_{2}, \gamma_{2}\right)\right\}}}(Y)$;

(2) $\overline{\overline{\widetilde{R}}_{\left\{\left(\alpha_{1}, \beta_{1}, \gamma_{1}\right)\right\}}}(Y) \subseteq \overline{\overline{\widetilde{R}_{\left\{\left(\alpha_{2}, \beta_{2}, \gamma_{2}\right)\right\}}}}(Y)$.

Proof. (1) Since $\alpha_{1} \leq \alpha_{2}, \beta_{1} \geq \beta_{2}$ and $\gamma_{1} \geq \gamma_{2}$, for any $x \in U$, we have

$$
\begin{aligned}
& \widetilde{R}_{\left\{\left(\alpha_{1}, \beta_{1}, \gamma_{1}\right)\right\}}(x)=\left\{y \in V \mid T_{\widetilde{R}}(x, y) \geq \alpha_{1}, I_{\widetilde{R}}(x, y) \leq \beta_{1}, F_{\widetilde{R}}(x, y) \leq \gamma_{1}\right\} \\
& \supseteq\left\{y \in V \mid T_{\widetilde{R}}(x, y) \geq \alpha_{2}, I_{\widetilde{R}}(x, y) \leq \beta_{2}, F_{\widetilde{R}}(x, y) \leq \gamma_{2}\right\} \\
& =\widetilde{R}_{\left\{\left(\alpha_{2}, \beta_{2}, \gamma_{2}\right)\right\}}(x) .
\end{aligned}
$$

By Definition 12, for any $x \in \widetilde{R}_{\left\{\left(\alpha_{1}, \beta_{1}, \gamma_{1}\right)\right\}}(Y)$, we have $\widetilde{R}_{\left\{\left(\alpha_{1}, \beta_{1}, \gamma_{1}\right)\right\}}(x) \subseteq Y$. Thus $\widetilde{R}_{\left\{\left(\alpha_{2}, \beta_{2}, \gamma_{2}\right)\right\}}(x) \subseteq \widetilde{R}_{\left\{\left(\alpha_{1}, \beta_{1}, \gamma_{1}\right)\right\}}(x) \subseteq Y$, which implies that $x \in \widetilde{\widetilde{R}}_{\left\{\left(\alpha_{2}, \beta_{2}, \gamma_{2}\right)\right\}}(Y)$. Hence, $\underline{\widetilde{R}_{\left\{\left(\alpha_{1}, \beta_{1}, \gamma_{1}\right)\right\}}}(Y) \subseteq \underline{\widetilde{R}_{\left\{\left(\alpha_{2}, \beta_{2}, \gamma_{2}\right)\right\}}}(Y)$.

So

(2) By (1), for any $x \in U$, we have $\widetilde{R}_{\left\{\left(\alpha_{1}, \beta_{1}, \gamma_{1}\right)\right\}}(x) \supseteq \widetilde{R}_{\left\{\left(\alpha_{2}, \beta_{2}, \gamma_{2}\right)\right\}}(x)$.

$$
\widetilde{R}_{\left\{\left(\alpha_{1}, \beta_{1}, \gamma_{1}\right)\right\}}(x) \cap Y \supseteq \widetilde{R}_{\left\{\left(\alpha_{2}, \beta_{2}, \gamma_{2}\right)\right\}}(x) \cap Y \text { for any } x \in U \text {. }
$$

By Definition 12, for any $x \in \overline{\widetilde{R}}_{\left\{\left(\alpha_{2}, \beta_{2}, \gamma_{2}\right)\right\}}(Y)$, we have $\widetilde{R}_{\left\{\left(\alpha_{2}, \beta_{2}, \gamma_{2}\right)\right\}}(x) \cap Y \neq \varnothing$. Thus, $\widetilde{R}_{\left\{\left(\alpha_{1}, \beta_{1}, \gamma_{1}\right)\right\}}(x) \cap Y \neq \varnothing$, which implies that $x \in \widetilde{R}_{\left\{\left(\alpha_{1}, \beta_{1}, \gamma_{1}\right)\right\}}(Y)$. Hence, $\widetilde{\widetilde{R}}_{\left\{\left(\alpha_{1}, \beta_{1}, \gamma_{1}\right)\right\}}(Y) \subseteq \widetilde{R}_{\left\{\left(\alpha_{2}, \beta_{2}, \gamma_{2}\right)\right\}}(Y)$.

Theorem 3. Let $\widetilde{R}, \widetilde{S}$ be two series single-valued neutrosophic relations from $U$ to $V$. If $\widetilde{R} \widetilde{\subseteq} \widetilde{S}$, then $\forall Y \subseteq V$, we have:

(1) $\quad \underline{\widetilde{S}_{\{(\alpha, \beta, \gamma)\}}}(Y) \subseteq \underline{\widetilde{R}_{\{(\alpha, \beta, \gamma)\}}}(Y)$; 
(2) $\quad \overline{\widetilde{R}_{\{(\alpha, \beta, \gamma)\}}}(Y) \subseteq \widetilde{\widetilde{S}}_{\{(\alpha, \beta, \gamma)\}}(Y)$.

Proof. (1) Since $\widetilde{R} \widetilde{\subseteq} \widetilde{S}$, we have

$$
\begin{aligned}
& T_{\widetilde{S}}(x, y) \geq T_{\widetilde{R}}(x, y), I_{\widetilde{S}}(x, y) \leq I_{\widetilde{R}}(x, y) \text { and } F_{\widetilde{S}}(x, y) \leq F_{\widetilde{R}}(x, y) \text { for any }(x, y) \in U \times V . \\
& \text { Then, } \widetilde{S}_{\{(\alpha, \beta, \gamma)\}}(x)=\left\{y \in V \mid T_{\widetilde{S}}(x, y) \geq \alpha, I_{\widetilde{S}}(x, y) \leq \beta, F_{\widetilde{S}}(x, y) \leq \gamma\right\} \\
& \supseteq\left\{y \in V \mid T_{\widetilde{R}}(x, y) \geq \alpha, I_{\widetilde{R}}(x, y) \leq \beta, F_{\widetilde{R}}(x, y) \leq \gamma\right\} \\
& =\widetilde{R}_{\{(\alpha, \beta, \gamma)\}}(x) .
\end{aligned}
$$

By Definition 12, for any $x \in \widetilde{S}_{\{(\alpha, \beta, \gamma)\}}(Y)$, we have $\widetilde{S}_{\{(\alpha, \beta, \gamma)\}}(x) \subseteq Y$. Thus, $\widetilde{R}_{\{(\alpha, \beta, \gamma)\}}(x) \subseteq \widetilde{S}_{\{(\alpha, \beta, \gamma)\}}(x) \subseteq Y$, which implies that $x \in \underline{\widetilde{R}}_{\{(\alpha, \beta, \gamma)\}}(Y)$. Hence, $\underline{\widetilde{S}_{\{(\alpha, \beta, \gamma)\}}}(Y) \subseteq \underline{\widetilde{R}_{\{(\alpha, \beta, \gamma)\}}}(Y)$.

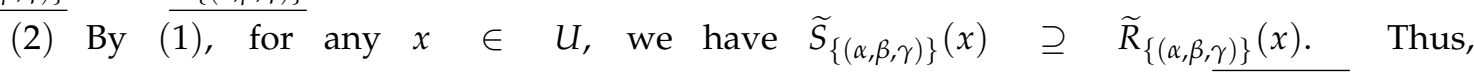
$\widetilde{R}_{\{(\alpha, \beta, \gamma)\}}(x) \cap Y \subseteq \widetilde{S}_{\{(\alpha, \beta, \gamma)\}}(x) \cap Y$ for any $x \in U$. By Definition 12, for any $x \in \widetilde{R}_{\{(\alpha, \beta, \gamma)\}}(Y)$, we have $\widetilde{R}_{\{(\alpha, \beta, \gamma)\}}(x) \cap Y \neq \varnothing$. Thus, $\widetilde{S}_{\{(\alpha, \beta, \gamma)\}}(x) \cap Y \neq \varnothing$, which implies that $x \in \widetilde{\widetilde{S}}_{\{(\alpha, \beta, \gamma)\}}(Y)$.

Hence, $\widetilde{R}_{\{(\alpha, \beta, \gamma)\}}(Y) \subseteq \widetilde{S}_{\{(\alpha, \beta, \gamma)\}}(Y)$.

Lemma 1. Let $\widetilde{R}, \widetilde{S}$ be two single-valued neutrosophic relations from $U$ to $V$. For any $x \in U$ and $\alpha, \beta, \gamma \in(0,1]$, we have:

(1) $\quad(\widetilde{R} \widetilde{\cup})_{\{(\alpha, \beta, \gamma)\}}(x) \supseteq \widetilde{R}_{\{(\alpha, \beta, \gamma)\}}(x) \cup \widetilde{S}_{\{(\alpha, \beta, \gamma)\}}(x)$;

(2) $\quad(\widetilde{R} \widetilde{\cap} \widetilde{S})_{\{(\alpha, \beta, \gamma)\}}(x)=\widetilde{R}_{\{(\alpha, \beta, \gamma)\}}(x) \cap \widetilde{S}_{\{(\alpha, \beta, \gamma)\}}(x)$.

Proof. (1) For any $x \in U$, we have:

$$
\begin{aligned}
& (\widetilde{R} \widetilde{\cup})_{\{(\alpha, \beta, \gamma)\}}(x)=\left\{y \in V \mid \max \left(T_{\widetilde{R}}(x, y), T_{\widetilde{S}}(x, y)\right) \geq \alpha, \min \left(I_{\widetilde{R}}(x, y), I_{\widetilde{S}}(x, y)\right) \leq \beta,\right. \\
& \left.\min \left(F_{\widetilde{R}}(x, y), F_{\widetilde{S}}(x, y)\right) \leq \gamma\right\} \\
& \supseteq\left\{y \in V \mid T_{\widetilde{R}}(x, y) \geq \alpha, I_{\widetilde{R}}(x, y) \leq \beta, F_{\widetilde{R}}(x, y) \leq \gamma\right\} \\
& \cup\left\{y \in V \mid T_{\widetilde{S}}(x, y) \geq \alpha, I_{\widetilde{S}}(x, y) \leq \beta, F_{\widetilde{S}}(x, y) \leq \gamma\right\} \\
& =\widetilde{R}_{\{(\alpha, \beta, \gamma)\}}(x) \cup \widetilde{S}_{\{(\alpha, \beta, \gamma)\}}(x)
\end{aligned}
$$

(2) For any $x \in U$, we have:

$$
\begin{gathered}
(\widetilde{R} \widetilde{\cap})_{\{(\alpha, \beta, \gamma)\}}(x)=\left\{y \in V \mid \min \left(T_{\widetilde{R}}(x, y), T_{\widetilde{S}}(x, y)\right) \geq \alpha, \max \left(I_{\widetilde{R}}(x, y), I_{\widetilde{S}}(x, y)\right) \leq \beta,\right. \\
\left.\quad \max \left(F_{\widetilde{R}}(x, y), F_{\widetilde{S}}(x, y)\right) \leq \gamma\right\} \\
=\left\{y \in V \mid T_{\widetilde{R}}(x, y) \geq \alpha, I_{\widetilde{R}}(x, y) \leq \beta, F_{\widetilde{R}}(x, y) \leq \gamma\right\} \\
\cap\left\{y \in V \mid T_{\widetilde{S}}(x, y) \geq \alpha, I_{\widetilde{S}}(x, y) \leq \beta, F_{\widetilde{S}}(x, y) \leq \gamma\right\} \\
=\widetilde{R}_{\{(\alpha, \beta, \gamma)\}}(x) \cap \widetilde{S}_{\{(\alpha, \beta, \gamma)\}}(x)
\end{gathered}
$$

Theorem 4. Let $\widetilde{R}, \widetilde{S}$ be two series single-valued neutrosophic relations from $U$ to $V$. For any $Y \subseteq V$ and $\alpha, \beta, \gamma \in(0,1]$, we have:

(1) $\quad \underline{(\widetilde{R} \widetilde{\widetilde{S}})_{\{(\alpha, \beta, \gamma)\}}}(Y) \subseteq \underline{\widetilde{R}_{\{(\alpha, \beta, \gamma)\}}}(Y) \cap \underline{\widetilde{S}_{\{(\alpha, \beta, \gamma)\}}}(Y) \subseteq \underline{\widetilde{R}_{\{(\alpha, \beta, \gamma)\}}}(Y) \cup \underline{\widetilde{S}_{\{(\alpha, \beta, \gamma)\}}}(Y) ;$

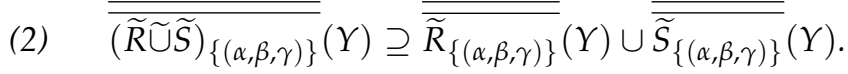

Proof. (1) By Lemma 1 (1), we have

$$
\underline{(\widetilde{R} \widetilde{\cup})_{\{(\alpha, \beta, \gamma)\}}}(Y)=\left\{x \in U \mid(\widetilde{R} \widetilde{\cup} \widetilde{S})_{\{(\alpha, \beta, \gamma)\}}(x) \subseteq Y\right\}
$$


So

$$
\begin{aligned}
& \subseteq\left\{x \in U \mid \widetilde{R}_{\{(\alpha, \beta, \gamma)\}}(x) \cup \widetilde{S}_{\{(\alpha, \beta, \gamma)\}}(x) \subseteq Y\right\} \\
& =\left\{x \in U \mid \widetilde{R}_{\{(\alpha, \beta, \gamma)\}}(x) \subseteq Y\right\} \cap\left\{x \in U \mid \widetilde{S}_{\{(\alpha, \beta, \gamma)\}}(x) \subseteq Y\right\} \\
& =\underline{\widetilde{R}}_{\{(\alpha, \beta, \gamma)\}}(Y) \cap \underline{\widetilde{S}_{\{(\alpha, \beta, \gamma)\}}}(Y) .
\end{aligned}
$$

$(\widetilde{R} \widetilde{\cup})_{\{(\alpha, \beta, \gamma)\}}(Y) \subseteq \underline{\widetilde{R}_{\{(\alpha, \beta, \gamma)\}}}(Y) \cap \underline{\widetilde{S}_{\{(\alpha, \beta, \gamma)\}}}(Y) \subseteq \underline{\widetilde{R}_{\{(\alpha, \beta, \gamma)\}}}(Y) \cup \underline{\widetilde{S}_{\{(\alpha, \beta, \gamma)\}}}(Y)$.

(2) By Lemma 1 (1), we have

$$
\begin{aligned}
\overline{(\widetilde{R} \widetilde{S})_{\{(\alpha, \beta, \gamma)\}}}(Y) & =\left\{x \in U \mid(\widetilde{R} \widetilde{S})_{\{(\alpha, \beta, \gamma)\}}(x) \cap Y \neq \varnothing\right\} \\
& \supseteq\left\{x \in U \mid\left(\widetilde{R}_{\{(\alpha, \beta, \gamma)\}}(x) \cup \widetilde{S}_{\{(\alpha, \beta, \gamma)\}}(x)\right) \cap Y \neq \varnothing\right\} \\
& =\left\{x \in U \mid \widetilde{R}_{\{(\alpha, \beta, \gamma)\}}(x) \cap Y \neq \varnothing\right\} \cup\left\{x \in U \mid \widetilde{S}_{\{(\alpha, \beta, \gamma)\}}(x) \cap Y \neq \varnothing\right\} \\
& =\widetilde{\widetilde{R}}_{\{(\alpha, \beta, \gamma)\}}(Y) \cup \widetilde{\widetilde{S}}_{\{(\alpha, \beta, \gamma)\}}(Y) .
\end{aligned}
$$

Theorem 5. Let $\widetilde{R}, \widetilde{S}$ be two series single-valued neutrosophic relations from $U$ to $V$. For any $Y \subseteq V$ and $\alpha, \beta, \gamma \in(0,1]$, we have:

(1) $\quad \underline{\underline{(\widetilde{R} \widetilde{\cap} \widetilde{S})_{\{(\alpha, \beta, \gamma)\}}}}(Y) \supseteq \underline{\underline{\widetilde{R}_{\{(\alpha, \beta, \gamma)\}}}}(Y) \cup \underline{\underline{\widetilde{S}_{\{(\alpha, \beta, \gamma)\}}}}(Y) \supseteq \underline{\widetilde{R}_{\{(\alpha, \beta, \gamma)\}}}(Y) \cap \underline{\widetilde{S}_{\{(\alpha, \beta, \gamma)\}}}(Y) ;$

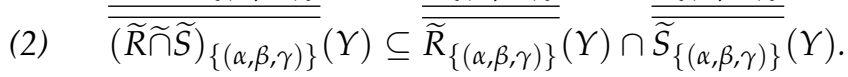

Proof. (1) By Lemma 1 (2), we have

$\underline{(\widetilde{R} \widetilde{\cap} \widetilde{S})_{\{(\alpha, \beta, \gamma)\}}}(Y)=\left\{x \in U \mid(\widetilde{R} \widetilde{\cap} \widetilde{S})_{\{(\alpha, \beta, \gamma)\}}(x) \subseteq Y\right\}$

$$
\begin{aligned}
& =\left\{x \in U \mid\left(\widetilde{R}_{\{(\alpha, \beta, \gamma)\}}(x) \cap \widetilde{S}_{\{(\alpha, \beta, \gamma)\}}(x)\right) \subseteq Y\right\} \\
& \supseteq\left\{x \in U \mid\left(\widetilde{R}_{\{(\alpha, \beta, \gamma)\}}(x) \subseteq Y\right\} \cup\left\{x \in U \mid\left(\widetilde{S}_{\{(\alpha, \beta, \gamma)\}}(x) \subseteq Y\right\}\right.\right. \\
& =\underline{\widetilde{R}_{\{(\alpha, \beta, \gamma)\}}}(Y) \cup \underline{\widetilde{S}_{\{(\alpha, \beta, \gamma)\}}}(Y) .
\end{aligned}
$$

Therefore,

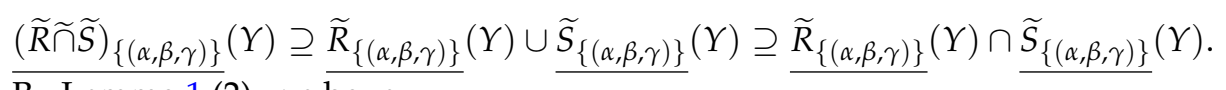

(2) By Lemma 1 (2), we have

$$
\begin{aligned}
\overline{(\widetilde{R} \widetilde{\cap})_{\{(\alpha, \beta, \gamma)\}}}(Y) & =\left\{x \in U \mid(\widetilde{R} \widetilde{\cap} \widetilde{S})_{\{(\alpha, \beta, \gamma)\}}(x) \cap Y \neq \varnothing\right\} \\
& =\left\{x \in U \mid\left(\widetilde{R}_{\{(\alpha, \beta, \gamma)\}} \cap \widetilde{S}_{\{(\alpha, \beta, \gamma)\}}\right)(x) \cap Y \neq \varnothing\right\} \\
& \subseteq\left\{x \in U \mid \widetilde{R}_{\{(\alpha, \beta, \gamma)\}}(x) \cap Y \neq \varnothing\right\} \cap\left\{x \in U \mid \widetilde{S}_{\{(\alpha, \beta, \gamma)\}}(x) \cap Y \neq \varnothing\right\} \\
& =\widetilde{\widetilde{R}}_{\{(\alpha, \beta, \gamma)\}}(Y) \cap \widetilde{\widetilde{S}}_{\{(\alpha, \beta, \gamma)\}}(Y) .
\end{aligned}
$$

Next, we define the inverse lower approximation and upper approximation on two universes w.r.t. $(U, V, \widetilde{R})$ and $(\alpha, \beta, \gamma)$ as follows:

Definition 13. Let $(U, V, \widetilde{R})$ be a generalized single-valued neutrosophic approximation space. For any $\alpha, \beta, \gamma \in(0,1], X \subseteq U$, the inverse lower approximation and upper approximation of $X$ on two universes w.r.t. $(U, V, \widetilde{R})$ and $(\alpha, \beta, \gamma)$ are defined as:

$$
\begin{aligned}
& \frac{\widetilde{R}_{\{(\alpha, \beta, \gamma)\}}^{-1}}{\widetilde{R}_{\{(\alpha, \beta, \gamma)\}}^{-1}}(X)=\left\{y \in V \mid \widetilde{R}_{\{(\alpha, \beta, \gamma)\}}^{-1}(y) \subseteq X \text { and } \widetilde{R}_{\{(\alpha, \beta, \gamma)\}}^{-1}(y) \neq \varnothing\right\}, \\
& =\left\{y \in V \mid \widetilde{R}_{\{(\alpha, \beta, \gamma)\}}^{-1}(y) \cap X \neq \varnothing \text { or } \widetilde{R}_{\{(\alpha, \beta, \gamma)\}}^{-1}(y)=\varnothing\right\} .
\end{aligned}
$$


The pair $\left.\underline{\left(\widetilde{R}_{\{(\alpha, \beta, \gamma)\}}^{-1}(X),\right.}, \widetilde{R}_{\{(\alpha, \beta, \gamma)\}}^{-1}(X)\right)$ is called the inverse rough set of $X$ w.r.t. $(U, V, \widetilde{R})$ and $(\alpha, \overline{\beta, \gamma})$.

Theorem 6. Let $(U, V, \widetilde{R})$ be a generalized single-valued neutrosophic approximation space. $\widetilde{R}_{\{(\alpha, \beta, \gamma)\}}$ is the $(\alpha, \beta, \gamma)$-cut relation given in Definition 10 from $U$ to $V$, where $\alpha, \beta, \gamma \in(0,1]$. For any $X, X_{1}, X_{2} \subseteq U$, we have:

(1) $\quad \widetilde{R}_{\{(\alpha, \beta, \gamma)\}}^{-1}(X) \subseteq \widetilde{R}_{\{(\alpha, \beta, \gamma)\}}^{-1}(X) ;$

(2) $\overline{\widetilde{R}_{\{(\alpha, \beta, \gamma)\}}^{-1}}(\varnothing)=\varnothing, \widetilde{R}_{\{(\alpha, \beta, \gamma)\}}^{-1}(U)=V$;

(3) $\quad \underline{\underline{\widetilde{R}_{\{(\alpha, \beta, \gamma)\}}^{-1}}}\left(X_{1} \cap X_{2}\right)=\underline{\widetilde{R}_{\{(\alpha, \beta, \gamma)\}}^{-1}}\left(X_{1}\right) \cap \underline{\widetilde{R}_{\{(\alpha, \beta, \gamma)\}}^{-1}}\left(X_{2}\right)$,

$$
\overline{\widetilde{R}_{\{(\alpha, \beta, \gamma)\}}^{-1}}\left(X_{1} \cup X_{2}\right)=\widetilde{R}_{\{(\alpha, \beta, \gamma)\}}^{-1}\left(X_{1}\right) \cup \widetilde{R}_{\{(\alpha, \beta, \gamma)\}}^{-1}\left(X_{2}\right) ;
$$

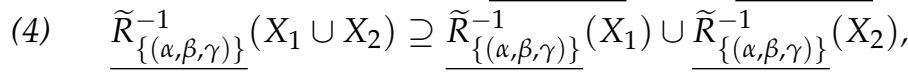

$$
\overline{\widetilde{R}_{\{(\alpha, \beta, \gamma)\}}^{-1}}\left(X_{1} \cap X_{2}\right) \subseteq \overline{\widetilde{R}_{\{(\alpha, \beta, \gamma)\}}^{-1}}\left(X_{1}\right) \cap \overline{\widetilde{R}_{\{(\alpha, \beta, \gamma)\}}^{-1}}\left(X_{2}\right) ;
$$

(5) If $X_{1} \subseteq X_{2}$, then $\underline{\widetilde{R}_{\{(\alpha, \beta, \gamma)\}}^{-1}}\left(X_{1}\right) \subseteq \underline{\widetilde{R}_{\{(\alpha, \beta, \gamma)\}}^{-1}}\left(X_{2}\right)$ and $\widetilde{R}_{\{(\alpha, \beta, \gamma)\}}^{-1}\left(X_{1}\right) \subseteq \widetilde{\widetilde{R}}_{\{(\alpha, \beta, \gamma)\}}^{-1}\left(X_{2}\right)$;

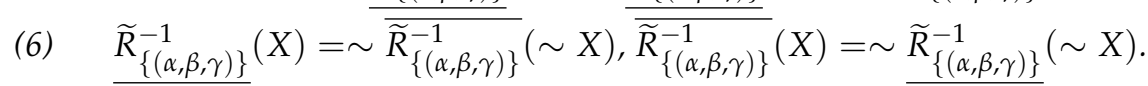

Proof. The proof is similar to that of Theorem 1.

Definition 14. Let $(U, V, \widetilde{R})$ be a generalized single-valued neutrosophic approximation space. $\widetilde{R}_{\{(\alpha, \beta, \gamma)\}}$ is $a(\alpha, \beta, \gamma)$-cut relation defined in Definition 10. For any $Y \subseteq V$, the approximate precision $\rho_{\widetilde{R}_{\{(\alpha, \beta, \gamma)\}}}(Y)$ of $Y$ w.r.t. $\widetilde{R}_{\{(\alpha, \beta, \gamma)\}}$ is defined as follows:

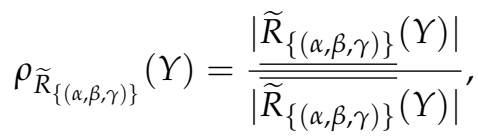

where $|Y|$ represents the cardinality of the set $Y$.

Let $\mu_{\widetilde{R}_{\{(\alpha, \beta, \gamma)\}}}(Y)=1-\rho_{\widetilde{R}_{\{(\alpha, \beta, \gamma)\}}}(Y)$, and $\mu_{\widetilde{R}_{\{(\alpha, \beta, \gamma)\}}}(Y)$ is called the rough degree of with regard to $\widetilde{R}_{\{(\alpha, \beta, \gamma)\}}$. It is obviously that $0 \leq \rho_{\widetilde{R}_{\{(\alpha, \beta, \gamma)\}}}(Y) \leq 1$ and $0 \leq \mu_{\widetilde{R}_{\{(\alpha, \beta, \gamma)\}}}(Y) \leq 1$.

The following Theorem 7 discusses the properties of approximation precision and rough degree.

Theorem 7. Let $(U, V, \widetilde{R})$ be a generalized single-valued neutrosophic approximation space. $\widetilde{R}_{\{(\alpha, \beta, \gamma)\}}$ is $a(\alpha, \beta, \gamma)$-cut relation defined in Definition 10. For any $Y_{1}, Y_{2} \subseteq V\left(Y_{1} \neq \varnothing, Y_{2} \neq \varnothing\right)$, then the rough degree and the approximate precision of the set $Y_{1}, Y_{2}, Y_{1} \cup Y_{2}$, and $Y_{1} \cap Y_{2}$ satisfy the following properties:

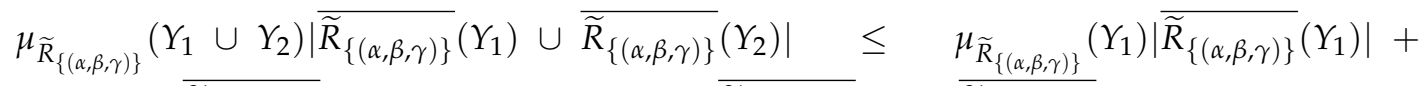

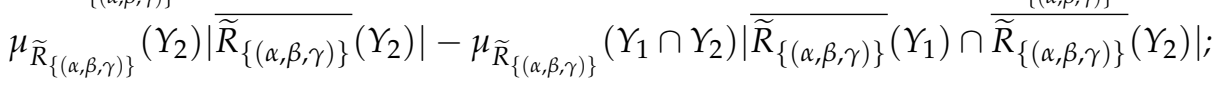

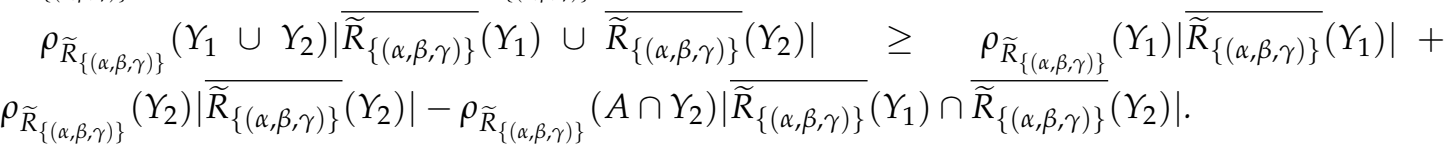

Proof. According to the definition of the rough degree, we have 


$$
\begin{aligned}
& \mu_{\widetilde{R}_{\{(\alpha, \beta, \gamma)\}}}\left(Y_{1} \cup Y_{2}\right)=1-\frac{\left|\widetilde{R}_{\{(\alpha, \beta, \gamma)\}}\left(Y_{1} \cup Y_{2}\right)\right|}{\left|\widetilde{\widetilde{R}}_{\{(\alpha, \beta, \gamma)\}}\left(Y_{1} \cup Y_{2}\right)\right|} \\
& =1-\frac{\left|\widetilde{R}_{\{(\alpha, \beta, \gamma)\}}\left(Y_{1} \cup Y_{2}\right)\right|}{\left|\widetilde{R}_{\{(\alpha, \beta, \gamma)\}}\left(Y_{1}\right) \cup \widetilde{R}_{\{(\alpha, \beta, \gamma)\}}\left(Y_{2}\right)\right|} \\
& \leq 1-\frac{\left|\widetilde{R}_{\{(\alpha, \beta, \gamma)\}}\left(Y_{1}\right) \cup \widetilde{R}_{\{(\alpha, \beta, \gamma)\}}\left(Y_{1}\right)\right|}{\left|\widetilde{\widetilde{R}}_{\{(\alpha, \beta, \gamma)\}}\left(Y_{1}\right) \cup \widetilde{\widetilde{R}}_{\{(\alpha, \beta, \gamma)\}}\left(Y_{2}\right)\right|} .
\end{aligned}
$$

Then, we have

$$
\begin{aligned}
& \mu_{\widetilde{R}_{\{(\alpha, \beta, \gamma)\}}}\left(Y_{1} \cup Y_{2}\right)\left|\overline{\widetilde{R}_{\{(\alpha, \beta, \gamma)\}}}\left(Y_{1}\right) \cup \overline{\widetilde{R}_{\{(\alpha, \beta, \gamma)\}}}\left(Y_{2}\right)\right| \\
& \leq\left|\overline{\widetilde{R}_{\{(\alpha, \beta, \gamma)\}}}\left(Y_{1}\right) \cup \overline{\widetilde{R}_{\{(\alpha, \beta, \gamma)\}}}\left(Y_{2}\right)\right|-\left|\underline{\mid \widetilde{R}_{\{(\alpha, \beta, \gamma)\}}}\left(Y_{1}\right) \cup \underline{\underline{\widetilde{R}_{\{(\alpha, \beta, \gamma)\}}}}\left(Y_{2}\right)\right| .
\end{aligned}
$$

Similarly, we have

$$
\begin{aligned}
& \mu_{\widetilde{R}_{\{(\alpha, \beta, \gamma)\}}}\left(Y_{1} \cap Y_{2}\right)=1-\frac{\left|\widetilde{R}_{\{(\alpha, \beta, \gamma)\}}\left(Y_{1} \cap Y_{2}\right)\right|}{\left|\widetilde{\widetilde{R}}_{\{(\alpha, \beta, \gamma)\}}\left(Y_{1} \cap Y_{2}\right)\right|} \\
& =1-\frac{\left|\widetilde{R}_{\{(\alpha, \beta, \gamma)\}}\left(Y_{1}\right) \cap \widetilde{R}_{\{(\alpha, \beta, \gamma)\}}\left(Y_{2}\right)\right|}{\left|\widetilde{R}_{\{(\alpha, \beta, \gamma \gamma)\}}\left(Y_{1} \cap Y_{2}\right)\right|} \\
& \leq 1-\frac{\left|\widetilde{R}_{\{(\alpha, \beta, \gamma \gamma)\}}\left(Y_{1}\right) \cap \widetilde{R}_{\{(\alpha, \beta, \gamma)\}}\left(Y_{1}\right)\right|}{\left|\widetilde{\widetilde{R}}_{\{(\alpha, \beta, \gamma)\}}\left(Y_{1}\right) \cup \widetilde{\widetilde{R}}_{\{(\alpha, \beta, \gamma)\}}\left(Y_{2}\right)\right|} .
\end{aligned}
$$

Hence,

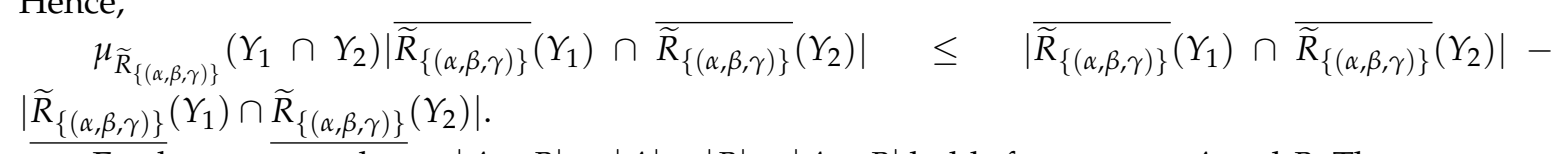

Furthermore, we know $|A \cup B|=|A|+|B|-|A \cap B|$ holds for any sets $A$ and $B$. Then,

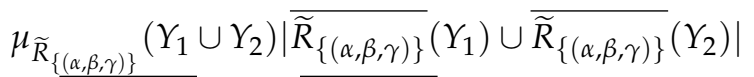

$$
\begin{aligned}
& \leq\left|\widetilde{\widetilde{R}}_{\{(\alpha, \beta, \gamma)\}}\left(Y_{1}\right) \cup \widetilde{\widetilde{R}}_{\{(\alpha, \beta, \gamma)\}}\left(Y_{2}\right)\right|-\left|\widetilde{R}_{\{(\alpha, \beta, \gamma)\}}\left(Y_{1}\right) \cup \widetilde{R}_{\{(\alpha, \beta, \gamma)\}}\left(Y_{2}\right)\right| \\
& =\left|\widetilde{\widetilde{R}}_{\{(\alpha, \beta, \gamma)\}}\left(Y_{1}\right)\right|+\left|\widetilde{R}_{\{(\alpha, \beta, \gamma)\}}\left(Y_{2}\right)\right|-\overline{\mid \widetilde{R}_{\{(\alpha, \beta, \gamma)\}}}\left(Y_{1}\right) \overline{\cap \widetilde{\widetilde{R}}_{\{(\alpha, \beta, \gamma)\}}}\left(Y_{2}\right)|-| \underline{\mid \widetilde{R}_{\{(\alpha, \beta, \gamma)\}}}\left(Y_{1}\right) \mid \\
& -\left|\underline{\underline{R_{\{(\alpha, \beta, \gamma)\}}}}\left(Y_{2}\right)\right|+\underline{\mid \widetilde{R}_{\{(\alpha, \beta, \gamma)\}}}\left(Y_{1}\right) \cap \underline{\widetilde{R}_{\{(\alpha, \beta, \gamma)\}}}\left(Y_{2}\right) \mid
\end{aligned}
$$

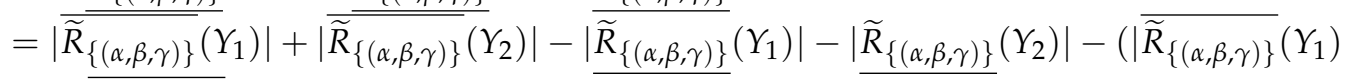

$$
\begin{aligned}
& \left.\cap \overline{\widetilde{R}_{\{(\alpha, \beta, \gamma)\}}}\left(Y_{2}\right)|-| \underline{\widetilde{R}_{\{(\alpha, \beta, \gamma)\}}}\left(Y_{1}\right) \cap \overline{\widetilde{R}_{\{(\alpha, \beta, \gamma)\}}}\left(Y_{2}\right) \mid\right)
\end{aligned}
$$

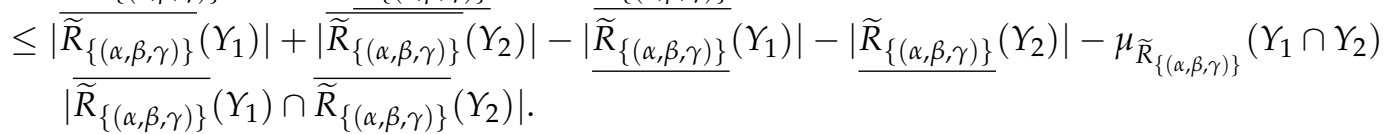

Furthermore, by

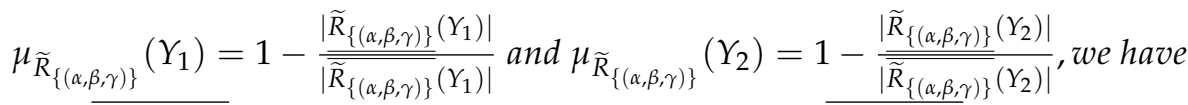

$$
\begin{aligned}
& \underline{\mid \widetilde{R}_{\{(\alpha, \beta, \gamma)\}}}\left(Y_{1}\right)\left|-\underline{\mid \widetilde{R}_{\{(\alpha, \beta, \gamma)\}}}\left(Y_{1}\right)\right|=\mu_{\widetilde{R}_{\{(\alpha, \beta, \gamma)\}}}\left(Y_{1}\right)\left|\overline{\widetilde{R}_{\{(\alpha, \beta, \gamma)\}}}\left(Y_{1}\right)\right| \text { and } \\
& \left|\overline{\widetilde{R}_{\{(\alpha, \beta, \gamma)\}}}\left(Y_{2}\right)\right|-\left|\underline{\mid \widetilde{R}_{\{(\alpha, \beta, \gamma)\}}}\left(Y_{2}\right)\right|=\mu_{\widetilde{R}_{\{(\alpha, \beta, \gamma)\}}}\left(Y_{2}\right)\left|\overline{\widetilde{R}}_{\{(\alpha, \beta, \gamma)\}}\left(Y_{2}\right)\right| \text {. }
\end{aligned}
$$


Therefore,

$$
\begin{aligned}
& \mu_{\widetilde{R}_{\{(\alpha, \beta, \gamma)\}}}\left(Y_{1} \cup Y_{2}\right)\left|\overline{\widetilde{R}_{\{(\alpha, \beta, \gamma)\}}}\left(Y_{1}\right) \cap \overline{\widetilde{R}_{\{(\alpha, \beta, \gamma)\}}}\left(Y_{2}\right)\right| \\
\leq & \left|\widetilde{R}_{\{(\alpha, \beta, \gamma)\}}\left(Y_{1}\right)\right|+\left|\widetilde{R}_{\{(\alpha, \beta, \gamma)\}}\left(Y_{2}\right)\right|-\left|\widetilde{R}_{\{(\alpha, \beta, \gamma)\}}\left(Y_{1}\right)\right|-\left|\widetilde{R}_{\{(\alpha, \beta, \gamma)\}}\left(Y_{2}\right)\right| \\
& -\mu_{\widetilde{R}_{\{(\alpha, \beta, \gamma)\}}}\left(Y_{1} \cap Y_{2}\right)\left|\widetilde{\widetilde{R}}_{\{(\alpha, \beta, \gamma)\}}\left(Y_{1}\right) \cap \widetilde{\widetilde{R}}_{\{(\alpha, \beta, \gamma)\}}\left(Y_{1}\right)\right| \\
= & \mu_{\widetilde{R}_{\{(\alpha, \beta, \gamma)\}}}\left(Y_{1}\right)\left|\widetilde{R}_{\{(\alpha, \beta, \gamma)\}}\left(Y_{1}\right)\right|+\mu_{\widetilde{R}_{\{(\alpha, \beta, \gamma)\}}\left(Y_{2}\right) \mid \widetilde{R}_{\{(\alpha, \beta, \gamma)\}}}\left(Y_{2}\right) \mid \\
& -\mu_{\widetilde{R}_{\{(\alpha, \beta, \gamma)\}}}\left(Y_{1} \cap Y_{2}\right)\left|\widetilde{R}_{\{(\alpha, \beta, \gamma)\}}\left(Y_{1}\right) \cap \widetilde{R}_{\{(\alpha, \beta, \gamma)\}}\left(Y_{2}\right)\right| .
\end{aligned}
$$

\section{Two Extended Models}

In this section, we give two extended rough set models of the model in Section 3, i.e., the degree rough set model and the variable precision rough set model.

\subsection{The Degree Rough Set Model on Two Different Universes}

Definition 15. Let $(U, V, \widetilde{R})$ be a generalized single-valued neutrosophic approximation space and $\widetilde{R}_{\{(\alpha, \beta, \gamma)\}}$ be $a(\alpha, \beta, \gamma)$-cut relation defined in Definition 10. For any $Y \subseteq V$, we define the degree lower and upper approximations of $Y$ w.r.t. the degree $k,(U, V, \widetilde{R})$ and $(\alpha, \beta, \gamma)$ as follows:

$$
\begin{aligned}
\underline{\widetilde{R}}_{\{(\alpha, \beta, \gamma)\}}^{k}(Y) & =\left\{x \in U|| \widetilde{R}_{\{(\alpha, \beta, \gamma)\}}(x)-Y \mid \leq k \text { and } \widetilde{R}_{\{(\alpha, \beta, \gamma)\}}(x) \neq \varnothing\right\} \\
& =\left\{x \in U|| \widetilde{R}_{\{(\alpha, \beta, \gamma)\}}(x)|-| \widetilde{R}_{\{(\alpha, \beta, \gamma)\}}(x) \cap Y \mid \leq k \text { and } \widetilde{R}_{\{(\alpha, \beta, \gamma)\}}(x) \neq \varnothing\right\} ; \\
\widetilde{\widetilde{R}}_{\{(\alpha, \beta, \gamma)\}} k & (Y)=\left\{x \in U|| \widetilde{R}_{\{(\alpha, \beta, \gamma)\}}(x) \cap Y \mid>\operatorname{kor} \widetilde{R}_{\{(\alpha, \beta, \gamma)\}}(x)=\varnothing\right\} .
\end{aligned}
$$

where $k$ is a finite nonnegative integer and $|Y|$ denotes the cardinality of the set $Y$.

The pair $\left.\underline{\widetilde{R}}_{\{(\alpha, \beta, \gamma)\}}^{k}(Y), \widetilde{\widetilde{R}}_{\{(\alpha, \beta, \gamma)\}}{ }^{k}(Y)\right)$ is called the degree rough set of $Y$ w.r.t. the degree $k$, $(U, V, \widetilde{R})$ and $(\overline{\alpha, \beta, \gamma})$.

We also define the positive region $\operatorname{pos}_{\widetilde{R}_{\{(\alpha, \beta, \gamma)\}}^{k}}(Y)$, negative region neg $\widetilde{R}_{\{(\alpha, \beta, \gamma)\}}^{k}(Y)$ and boundary region $\operatorname{bn}_{\widetilde{R}_{\{(\alpha, \beta, \gamma)\}}^{k}(Y)}$ of $Y$ as follows:

$$
\begin{aligned}
& \operatorname{pos}_{\widetilde{R}_{\{(\alpha, \beta, \gamma)\}}^{k}}(Y)=\underline{\widetilde{R}}_{\{(\alpha, \beta, \gamma)\}}{ }^{k}(Y), \\
& \operatorname{neg}_{\widetilde{R}_{\{(\alpha, \beta, \gamma)\}}^{k}}(Y)=U-\widetilde{\widetilde{R}}_{\{(\alpha, \beta, \gamma)\}}(Y), \\
& \operatorname{bn}_{\widetilde{R}_{\{(\alpha, \beta, \gamma)\}}^{k}}(Y)=\widetilde{\widetilde{R}}_{\{(\alpha, \beta, \gamma)\}}{ }^{k}(Y)-\underline{\widetilde{R}_{\{(\alpha, \beta, \gamma)\}}}{ }^{k}(Y) .
\end{aligned}
$$

Remark 4. In Definition 15, if $k=0$, then

$$
\begin{aligned}
\widetilde{\widetilde{R}}_{\{(\alpha, \beta, \gamma)\}}{ }^{0}(Y) & =\left\{x \in U|| \widetilde{R}_{\{(\alpha, \beta, \gamma)\}}(x)-Y \mid \leq 0 \text { and } \widetilde{R}_{\{(\alpha, \beta, \gamma)\}}(x) \neq \varnothing\right\} \\
& =\left\{x \in U|| \widetilde{R}_{\{(\alpha, \beta, \gamma)\}}(x)-Y \mid=0 \text { and } \widetilde{R}_{\{(\alpha, \beta, \gamma)\}}(x) \neq \varnothing\right\} \\
& =\left\{x \in U \mid \widetilde{R}_{\{(\alpha, \beta, \gamma)\}}(x) \subseteq Y \text { and } \widetilde{R}_{\{(\alpha, \beta, \gamma)\}}(x) \neq \varnothing\right\} \\
& =\widetilde{R}_{\{(\alpha, \beta, \gamma)\}}(Y) ; \\
\widetilde{\widetilde{R}}_{\{(\alpha, \beta, \gamma)\}}(Y) & =\left\{x \in U|| \widetilde{R}_{\{(\alpha, \beta, \gamma)\}}(x) \cap Y \mid>0 \text { or } \widetilde{R}_{\{(\alpha, \beta, \gamma)\}}(x)=\varnothing\right\} \\
& =\left\{x \in U \mid \widetilde{R}_{\{(\alpha, \beta, \gamma)\}}(x) \cap Y \neq \varnothing \text { or } \widetilde{R}_{\{(\alpha, \beta, \gamma)\}}(x)=\varnothing\right\} \\
& =\widetilde{\widetilde{R}}_{\{(\alpha, \beta, \gamma)\}}(Y),
\end{aligned}
$$

which implies that the lower and upper approximation operators in Definition 12 are special cases of the degree lower and upper approximation operators in Definition 15, respectively. 
In following Theorem 8 , we discuss some properties of the degree lower and upper approximation operators.

Theorem 8. Let $(U, V, \widetilde{R})$ be a generalized single-valued neutrosophic approximation space, and $\widetilde{R}_{\{(\alpha, \beta, \gamma)\}}$ is the $(\alpha, \beta, \gamma)$-cut relation defined in Definition 10. For any $Y, Y_{1}, Y_{2} \subseteq V$, we have:

$$
\begin{aligned}
& \underline{\widetilde{R}_{\{(\alpha, \beta, \gamma)\}}}{ }^{0}\left(Y_{1} \cap Y_{2}\right)=\underline{\widetilde{R}_{\{(\alpha, \beta, \gamma)\}}}\left(Y_{1}\right) \cap \underline{\widetilde{R}_{\{(\alpha, \beta, \gamma)\}}}{ }^{0}\left(Y_{2}\right), \\
& {\overline{\widetilde{R}_{\{(\alpha, \beta, \gamma)\}}}}^{0}\left(Y_{1} \cup Y_{2}\right)={\overline{\widetilde{R}_{\{(\alpha, \beta, \gamma)\}}}}^{0}\left(Y _ { 1 } \cup { \overline { \widetilde { R } _ { \{ ( \alpha , \beta , \gamma ) \} } } } ^ { 0 } \left(Y_{2} ;\right.\right.
\end{aligned}
$$

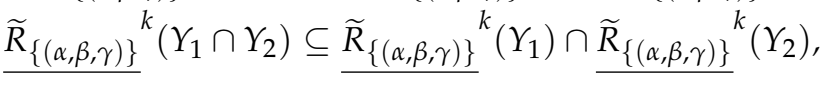

$$
\begin{aligned}
& \overline{\widetilde{R}}_{\{(\alpha, \beta, \gamma)\}}{ }^{k}\left(Y_{1} \cup Y_{2}\right) \supseteq{\overline{\widetilde{R}_{\{(\alpha, \beta, \gamma)\}}}}^{k}\left(Y_{1}\right) \cup{\widetilde{\widetilde{R}_{\{(\alpha, \beta, \gamma)\}}}}^{k}\left(Y_{2}\right) ;
\end{aligned}
$$

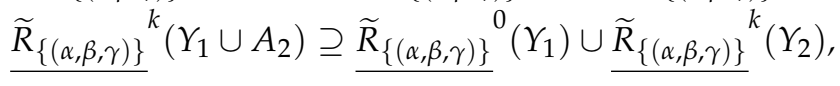

$$
\begin{aligned}
& {\widetilde{\widetilde{R}_{\{(\alpha, \beta, \gamma)\}}}}^{k}\left(Y_{1} \cap Y_{2}\right) \subseteq{\widetilde{\widetilde{R}_{\{(\alpha, \beta, \gamma)\}}}}^{k}\left(Y_{1}\right) \cap{\widetilde{\widetilde{R}_{\{(\alpha, \beta, \gamma)\}}}}^{k}\left(Y_{2}\right) ;
\end{aligned}
$$

(4) $\quad \underline{\widetilde{R}}_{\{(\alpha, \beta, \gamma)\}}{ }^{k}(Y)=\sim \widetilde{\widetilde{R}}_{\{(\alpha, \beta, \gamma)\}}{ }^{k}(\sim Y)$,

$$
\widetilde{\widetilde{R}}_{\{(\alpha, \beta, \gamma)\}}{ }^{k}(Y)=\sim \underline{\widetilde{R}}_{\{(\alpha, \beta, \gamma)\}}^{k}(\sim Y) ;
$$

(5) If $Y_{1} \subseteq Y_{2}$, then $\underline{\widetilde{R}}_{\{(\alpha, \beta, \gamma)\}}{ }^{k}\left(Y_{1}\right) \subseteq \underline{\widetilde{R}}_{\{(\alpha, \beta, \gamma)\}}{ }^{k}\left(Y_{2}\right)$ and $\overline{\widetilde{R}}_{\{(\alpha, \beta, \gamma)\}}{ }^{k}\left(Y_{1}\right) \subseteq \overline{\widetilde{R}}_{\{(\alpha, \beta, \gamma)\}}{ }^{k}\left(Y_{2}\right)$;

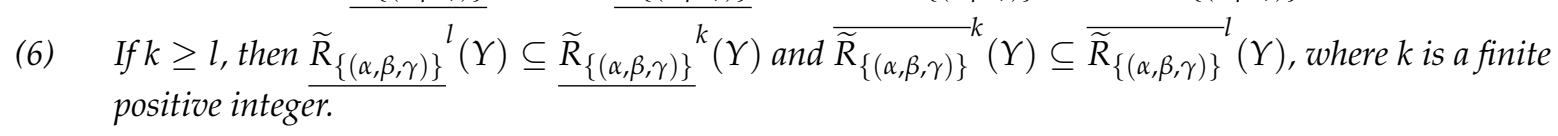

Proof. We only prove (4) and (6).

(4) Notice that $Y_{1}-Y_{2}=Y_{1} \cap\left(\sim Y_{2}\right)$ and $\sim(\sim Y)=Y$ for any set $Y \in V$; we have

$$
\begin{aligned}
\sim_{\{(\alpha, \beta, \gamma)\}} \widetilde{\widetilde{R}}^{k}(\sim Y) & =\sim\left\{x \in U|| \widetilde{R}_{\{(\alpha, \beta, \gamma)\}}(x) \cap(\sim Y) \mid>k \text { or } \widetilde{R}_{\{(\alpha, \beta, \gamma)\}}(x)=\varnothing\right\} \\
& =\sim\left\{x \in U|| \widetilde{R}_{\{(\alpha, \beta, \gamma)\}}(x)-Y \mid>k \text { or } \widetilde{R}_{\{(\alpha, \beta, \gamma)\}}(x)=\varnothing\right\} \\
& =\left\{x \in U|| \widetilde{R}_{\{(\alpha, \beta, \gamma)\}}(x) \cap(\sim Y) \mid \leq k \text { and } \widetilde{R}_{\{(\alpha, \beta, \gamma)\}}(x) \neq \varnothing\right\} \\
& =\widetilde{R}_{\{(\alpha, \beta, \gamma)\}}{ }^{k}(Y), \\
\sim \widetilde{\widetilde{R}}_{\{(\alpha, \beta, \gamma)\}}{ }^{k}(\sim Y) & =\sim\left\{x \in U|| \widetilde{R}_{\{(\alpha, \beta, \gamma)\}}(x)-(\sim Y) \mid \leq k \text { and } \widetilde{R}_{\{(\alpha, \beta, \gamma)\}}(x) \neq \varnothing\right\} \\
& =\sim\left\{x \in U|| \widetilde{R}_{\{(\alpha, \beta, \gamma)\}}(x) \cap(Y) \mid \leq k \text { and } \widetilde{R}_{\{(\alpha, \beta, \gamma)\}}(x) \neq \varnothing\right\} \\
& =\left\{x \in U|| \widetilde{R}_{\{(\alpha, \beta, \gamma)\}}(x) \cap(Y) \mid>k \text { or } \widetilde{R}_{\{(\alpha, \beta, \gamma)\}}(x)=\varnothing\right\} \\
& =\widetilde{\widetilde{R}}_{\{(\alpha, \beta, \gamma)\}}{ }^{k}(Y) .
\end{aligned}
$$

(6) Since $k \geq l$, for any $x \in U$ and $Y \subseteq V$, we have

$$
\begin{aligned}
\underline{\widetilde{R}_{\{(\alpha, \beta, \gamma)\}}^{l}(Y)} & =\left\{x \in U \| \widetilde{R}_{\{(\alpha, \beta, \gamma)\}}(x)-Y \mid \leq l \text { and } \widetilde{R}_{\{(\alpha, \beta, \gamma)\}}(x) \neq \varnothing\right\} \\
& \subseteq\left\{x \in U \| \widetilde{R}_{\{(\alpha, \beta, \gamma)\}}(x)-Y \mid \leq k \text { and } \widetilde{R}_{\{(\alpha, \beta, \gamma)\}}(x) \neq \varnothing\right\} \\
& =\widetilde{\widetilde{R}}_{\{(\alpha, \beta, \gamma)\}}{ }^{k}(Y) .
\end{aligned}
$$

For any $x \in \frac{\widetilde{R}_{\{(\alpha, \beta, \gamma)\}} l}{\widetilde{R}}(Y)$, we have $\left|\widetilde{R}_{\{(\alpha, \beta, \gamma)\}}(x)-Y\right| \leq l$ and $\widetilde{R}_{\{(\alpha, \beta, \gamma)\}}(x) \neq \varnothing$, then we have $\left|\widetilde{R}_{\{(\alpha, \beta, \gamma)\}}(x)-Y\right| \leq k$ and $\widetilde{R}_{\{(\alpha, \beta, \gamma)\}}(x) \neq \varnothing$, so $x \in \underline{\widetilde{R}}_{\{(\alpha, \beta, \gamma)\}}{ }^{k}(Y)$. Hence, $\underline{\widetilde{R}}_{\{(\alpha, \beta, \gamma)\}}^{l}(Y) \subseteq \underline{\widetilde{R}}_{\{(\alpha, \beta, \gamma)\}}{ }^{k}(Y)$.

Remark 5. In general, $\widetilde{R}_{\{(\alpha, \beta, \gamma)\}}{ }^{k}\left(Y_{1} \cap Y_{2}\right) \supseteq{\widetilde{\widetilde{R}_{\{(\alpha, \beta, \gamma)\}}}}^{k}\left(Y_{1}\right) \cap{\widetilde{\widetilde{R}_{\{(\alpha, \beta, \gamma)\}}}}^{k}\left(Y_{2}\right)$ does not hold, where $k$ is a finite positive integer. The reason is that if $k>0$, then $\left|\widetilde{R}_{\{(\alpha, \beta, \gamma)\}}-Y_{1}\right| \leq k$ and $\left|\widetilde{R}_{\{(\alpha, \beta, \gamma)\}}-Y_{2}\right| \leq$ $k \neq\left|\widetilde{R}_{\{(\alpha, \beta, \gamma)\}}-Y_{1} \cap Y_{2}\right| \leq k$. Besides, $\overline{\widetilde{R}}_{\{(\alpha, \beta, \gamma)\}}{ }^{k}\left(Y_{1} \cup Y_{2}\right) \subseteq{\widetilde{\widetilde{R}_{\{(\alpha, \beta, \gamma)\}}}}^{k}\left(Y_{1}\right) \cup{\underline{\widetilde{R}_{\{(\alpha, \beta, \gamma)\}}}}^{k}\left(Y_{2}\right)$ 
also does not hold in general, where $k$ is a finite positive integer. The reason is that if $k>0$, then $\left|\widetilde{R}_{\{(\alpha, \beta, \gamma)\}}(x) \cap\left(Y_{1} \cup Y_{2}\right)\right|>k \neq\left|\widetilde{R}_{\{(\alpha, \beta, \gamma)\}} \cap Y_{1}\right|>k$ or $\left|\widetilde{R}_{\{(\alpha, \beta, \gamma)\}} \cap Y_{2}\right|>k$.

\subsection{The Variable Precision Rough Set Model on Two Different Universes}

Definition 16. Let $(U, V, \widetilde{R})$ be a generalized single valued neutrosophic approximation space. For any $Y \subseteq V, \alpha, \beta, \gamma \in(0,1]$, we define the variable precision lower and upper approximation of $Y$ w.r.t. the control parameter $v,(U, V, \widetilde{R})$ and $(\alpha, \beta, \gamma)$ as follows, respectively:

$$
\begin{aligned}
& \frac{V \widetilde{R}_{\{(\alpha, \beta, \gamma)\}}}{v}(Y)=\left\{x \in U \mid \frac{\left|\widetilde{R}_{\{\alpha, \beta, \gamma \gamma\}}(x) \cap Y\right|}{\left|\widetilde{R}_{\{(\alpha, \beta, \gamma)\}}(x)\right|} \geq 1-v, \widetilde{R}_{\{(\alpha, \beta, \gamma)\}}(x) \neq \varnothing\right\}, \\
& \overline{V \widetilde{R}}_{\{(\alpha, \beta, \gamma)\}}(Y)=\left\{x \in U \mid \frac{\left|\widetilde{R}_{\{(\alpha, \beta, \gamma)\}}(x) \cap Y\right|}{\left|\widetilde{R}_{\{(\alpha, \beta, \gamma)\}}(x)\right|}>v, \widetilde{R}_{\{(\alpha, \beta, \gamma)\}}(x) \neq \varnothing\right\} \cup\left\{x \in U \mid \widetilde{R}_{\{(\alpha, \beta, \gamma)\}}(x)=\varnothing\right\},
\end{aligned}
$$

where $v \in[0,0.5),|Y|$ denotes the cardinality of the set $Y$.

The pair $\left(V \widetilde{R}_{\{(\alpha, \beta, \gamma)\}}{ }^{v},{\overline{V \widetilde{R}_{\{(\alpha, \beta, \gamma)\}}}}^{v}\right)$ is called the variable precision single-valued neutrosophic rough set of $A$ with regard to the control parameter $v,(U, V, \widetilde{R})$ and $(\alpha, \beta, \gamma)$.

We also define the positive region $\operatorname{pos}_{V \widetilde{R}_{\{(\alpha, \beta, \gamma)\}}^{v}}(Y)$, negative region neg $\widetilde{R}_{\{(\alpha, \beta, \gamma)\}}^{v}(Y)$ and boundary region $\operatorname{bn}_{V \widetilde{R}_{\{(\alpha, \beta, \gamma)\}}^{v}}(Y)$ of $Y$ about $\widetilde{R}_{\{(\alpha, \beta, \gamma)\}}^{v}(Y)$ as follows:

$$
\begin{aligned}
& \operatorname{pos}_{V \widetilde{R}_{\{(\alpha, \beta, \gamma)\}}^{v}}(Y)=\underline{V \widetilde{R}_{\{(\alpha, \beta, \gamma)\}}}{ }^{v}(Y), \\
& \operatorname{neg}_{V \widetilde{R}_{\{(\alpha, \beta, \gamma)\}}^{v}}(Y)=U-{\overline{V \widetilde{R}_{\{(\alpha, \beta, \gamma)\}}}}^{v}(Y) \text {, } \\
& \operatorname{bn}_{V \widetilde{R}_{\{(\alpha, \beta, \gamma)\}}^{v}}(Y)={\overline{V \widetilde{R}_{\{(\alpha, \beta, \gamma)\}}}}^{v}(Y)-\underline{V \widetilde{R}_{\{(\alpha, \beta, \gamma)\}}}{ }^{v}(Y) .
\end{aligned}
$$

The following Theorems 9 and 10 can be easily obtained by Definition 16 .

Theorem 9. Let $(U, V, \widetilde{R})$ be a generalized single-valued neutrosophic approximation space. For any $Y \subseteq V$, $\alpha, \beta, \gamma \in[0,1], v=[0,0.5)$, then:

(1) $\frac{\widetilde{R}_{\{(\alpha, \beta, \gamma)\}}(Y) \subseteq V \widetilde{R}_{\{(\alpha, \beta, \gamma)\}}^{v}}{\overline{V \widetilde{R}}_{\{(\alpha, \beta, \gamma)\}}(Y) \text {; }}(Y) \subseteq \widetilde{R}_{\{(\alpha, \beta, \gamma)\}}(Y)$.

Proof. The proof is straightforward from Definition 16.

Remark 6. In Theorem 9, if $v=0$, then:

$$
\text { (1) } \frac{V \widetilde{R}_{\{(\alpha, \beta, \gamma)\}}{ }^{0}(Y)=\widetilde{R}_{\{(\alpha, \beta, \gamma)\}}}{\overline{V \widetilde{R}}_{\{(\alpha, \beta, \gamma)\}}}(Y) \text {; }
$$

Theorem 10. Let $(U, V, \widetilde{R})$ be a generalized single-valued neutrosophic approximation space. For any $Y \subseteq V$, $\alpha, \beta, \gamma \in[0,1]$. Then:

$$
\begin{aligned}
& \text { (1) } \quad{\underline{V \widetilde{R}_{\{(\alpha, \beta, \gamma)\}}}}^{0.5}(Y)=\bigcup_{v \in[0,0.5)} \underline{V \widetilde{R}_{\{(\alpha, \beta, \gamma)\}}}{ }^{v}(Y) \text {; } \\
& \text { (2) }{\overline{V \widetilde{R}_{\{(\alpha, \beta, \gamma)\}}}}^{0.5}(Y)=\bigcap_{v \in[0,0.5)}{\overline{V \widetilde{R}_{\{(\alpha, \beta, \gamma)\}}}}^{v}(Y) \text {. }
\end{aligned}
$$

Proof. The proof is straightforward from Definition 16.

\section{An Application in Multi-Attribute Decision Making}

In this section, we illustrate the application of the rough set model in generalized single-valued neutrosophic spaces proposed in Section 3. 
We present the medical decision procedure based on the proposed rough set model in a single-valued neutrosophic environment as follows.

Assume that the universe $U=\left\{x_{1}, x_{2}, x_{3}, \cdots, x_{m}\right\}$ denotes a set of patients and the universe $V=\left\{x_{1}, x_{2}, x_{3}, \cdots, x_{n}\right\}$ denotes a set of symptoms. Let $\widetilde{R}$ be an SVNR from $U$ to $V$. For any $\left(x_{i}, y_{j}\right) \in U \times V, T_{\widetilde{R}}\left(x_{i}, y_{j}\right), I_{\widetilde{R}}\left(x_{i}, y_{j}\right), F_{\widetilde{R}}\left(x_{i}, y_{j}\right)$ represent the truth membership degree, indeterminacy membership degree and falsity membership degree of patient $x_{i}$ with symptoms $y_{j}$, respectively. Given a patient $x_{i}$, the doctor can present the relationship degree decreased by a single-valued neutrosophic number, i.e., $\widetilde{R}\left(x_{i}, y_{j}\right)$ between the patient $x_{i}$ and the symptom $y_{j}$. The $(\alpha, \beta, \gamma)$ is given in advance by another doctor and represents the doctors' lowest requirements on the membership degree. For any $Y \subseteq V, Y$ denotes a certain disease and has the basic symptoms $y_{j} \in Y$. For a patient $x_{i}$, if $x_{i} \in \widetilde{R}_{\{(\alpha, \beta, \gamma)\}}(Y)$, then we can be sure that the patient $x_{i}$ is suffering from the disease $Y$ and must receive treatment immediately; if $x_{i} \in \widetilde{R}_{\{(\alpha, \beta, \gamma)\}}(Y)-\widetilde{R}_{\{(\alpha, \beta, \gamma)\}}(Y)$, we cannot be sure whether the patient $x_{i}$ is suffering from the disease $Y$ or not. Therefore, the doctor needs to carry out a second choice to decide whether the patient $x_{i}$ is suffering from the disease $Y$ or not; if $x_{i} \in U-\widetilde{R}_{\{(\alpha, \beta, \gamma)\}}(Y)$, then we can be sure that the patient $x_{i}$ is not suffering from the disease $Y$ and does not need to receive treatment.

Next, we give an example to illustrate the method above.

Example 2. Let $U=\left\{x_{1}, x_{2}, x_{3}, x_{4}, x_{5}\right\}$ be five patients, $V=\left\{y_{1}, y_{2}, y_{3}, y_{4}, y_{5}, y_{6}, y_{7}\right\}$ be seven symptoms, where $y_{j}(j=1,2,3,4,5,6,7)$ stand for "tired", "a stuffed-up nose", "headache", "chill", "stomach pain", "dry cough" and "chest-pain". $\widetilde{R}$ is the medical knowledge statistic data of the relationship of the patient $x_{i}\left(x_{i} \in U\right)$ and the symptom $y_{j}\left(y_{j} \in V\right.$ ), and $\widetilde{R}$ is an SVNR from $U$ to $V$ (given in Table 2). For any $\left(x_{i}, y_{j}\right) \in U \times V$, $T_{\widetilde{R}}\left(x_{i}, y_{j}\right), I_{\widetilde{R}}\left(x_{i}, y_{j}\right), F_{\widetilde{R}}\left(x_{i}, y_{j}\right)$ represent the truth membership degree, indeterminacy membership degree and falsity membership degree of patient $x_{i}$ with symptoms $y_{j}$, respectively. For example, $\widetilde{R}\left(x_{1}, y_{1}\right)=(0.2,0.1,0.8)$ indicates that the truth membership, indeterminacy membership and falsity membership of patient $x_{1}$ with symptoms $y_{1}$ is $0.2,0.1,0.8$, respectively.

Table 2. The single-valued neutrosophic relation $\widetilde{R}$ of the symptoms and patients.

\begin{tabular}{cccccccc}
\hline$\widetilde{\boldsymbol{R}}$ & $y_{\mathbf{1}}$ & $\boldsymbol{y}_{\mathbf{2}}$ & $y_{\mathbf{3}}$ & $y_{\mathbf{4}}$ & $y_{5}$ & $y_{\mathbf{6}}$ & $y_{7}$ \\
\hline$x_{1}$ & $(0.6,0,1,0.4)$ & $(1,0,0)$ & $(0.6,0.2,0.2)$ & $(0.8,0.3,0.2)$ & $(0,0,1)$ & $(0.9,0.1,0.2)$ & $(0.1,0.1,0.9)$ \\
$x_{2}$ & $(1,0.2,0)$ & $(0,0,1)$ & $(0.8,0.1,0.1)$ & $(0.1,0.1,1.7)$ & $(0,0,1)$ & $(0.8,0.2,0.1)$ & $(0.2,0.1,0.6)$ \\
$x_{3}$ & $(0.8,0.1,0.5)$ & $(0,0.3,1)$ & $(0.2,0.2,0.8)$ & $(0.2,0.1,0.8)$ & $(0.7,0.1,0.2)$ & $(0.1,0.2,0.8)$ & $(1,0,0)$ \\
$x_{4}$ & $(1,0.3,0.1)$ & $(0,0,1)$ & $(0.3,0.1,0.7)$ & $(0,0,1)$ & $(0,0,1)$ & $(0.7,0.3,0.2)$ & $(0,0,1)$ \\
$x_{5}$ & $(0.1,0.2,0.7)$ & $(0,0,1)$ & $(0,0.2,0.9)$ & $(0.2,0.1,0.7)$ & $(1,0,0)$ & $(0,0,1)$ & $(0.7,0.3,0.2)$ \\
\hline
\end{tabular}

Let $Y=\left\{y_{1}, y_{2}, y_{3}, y_{6}\right\} \subseteq V$ denote a certain disease showing four basic symptoms in the clinic.

Case 1. Take $(\alpha, \beta, \gamma)=(0.5,0.5,0.5)$; by Definition 10 , we can get the cut relation $\widetilde{R}_{\{(0.5,0.5,0.5)\}}$ (given in Table 3).

Table 3. The cut relation $\widetilde{R}_{\{(0.5,0.5,0.5)\}}$.

\begin{tabular}{cccccccc}
\hline$\widetilde{\boldsymbol{R}}_{\{(\mathbf{0 . 5}, \mathbf{0 . 5 , 0 . 5 ) \}}}$ & $\boldsymbol{y}_{\mathbf{1}}$ & $\boldsymbol{y}_{\mathbf{2}}$ & $\boldsymbol{y}_{\mathbf{3}}$ & $\boldsymbol{y}_{\mathbf{4}}$ & $\boldsymbol{y}_{\mathbf{5}}$ & $\boldsymbol{y}_{\mathbf{6}}$ & $\boldsymbol{y}_{\mathbf{7}}$ \\
\hline$x_{1}$ & 1 & 1 & 1 & 1 & 0 & 1 & 0 \\
$x_{2}$ & 1 & 0 & 1 & 0 & 0 & 1 & 0 \\
$x_{3}$ & 1 & 0 & 0 & 0 & 1 & 0 & 1 \\
$x_{4}$ & 1 & 0 & 0 & 0 & 0 & 1 & 0 \\
$x_{5}$ & 0 & 0 & 0 & 0 & 1 & 0 & 1 \\
\hline
\end{tabular}

According to Definition 11, we can get

$\widetilde{R}_{\{(0.5,0.5,0.5)\}}\left(x_{1}\right)=\left\{y_{1}, y_{2}, y_{3}, y_{4}, y_{6}\right\}$, 


$$
\begin{aligned}
& \widetilde{R}_{\{(0.5,0.5,0.5)\}}\left(x_{2}\right)=\left\{y_{1}, y_{3}, y_{6}\right\}, \\
& \widetilde{R}_{\{(0.5,0.5,0.5)\}}\left(x_{3}\right)=\left\{y_{1}, y_{5}, y_{7}\right\}, \\
& \widetilde{R}_{\{(0.5,0.5,0.5)\}}\left(x_{4}\right)=\left\{y_{1}, y_{6}\right\}, \\
& \widetilde{R}_{\{(0.5,0.5,0.5)\}}\left(x_{5}\right)=\left\{y_{5}, y_{7}\right\} .
\end{aligned}
$$

Then, by Definition 12, we can calculate the lower approximation, the upper approximation, the boundary region and the negative region of $Y$ as follows, respectively.

$$
\begin{aligned}
& \frac{\widetilde{R}_{\{(0.5,0.5,0.5)\}}(Y)=\left\{x_{2}, x_{4}\right\},}{\widetilde{R}_{\{(0.5,0.5,0.5)\}}(Y)=\left\{x_{1}, x_{2}, x_{3}, x_{4}\right\},} \\
& b n_{\widetilde{R}_{\{(0.5,0.0,0.5)\}}}(Y)=\left\{x_{1}, x_{3}\right\}, \\
& n e g_{\widetilde{R}_{\{(0.5,0.5,0.5)\}}}(Y)=\left\{x_{5}\right\} .
\end{aligned}
$$

By Definition 14, we also can calculate the approximate precision of the set $Y(Y \subseteq V)$ as follows:

$\rho_{\widetilde{R}_{\{(\alpha, \beta, \gamma)\}}}(Y)=\frac{1}{2}$.

Thus, we can obtain the following conclusions:

(1) Patients $x_{2}$ and $x_{4}$ are suffering from the disease $Y$ and must receive treatment immediately.

(2) We cannot determine whether patients $x_{1}$ and $x_{3}$ are suffering from the disease $Y$ or not.

(3) The patient $x_{5}$ is not suffering from the disease $Y$ and does not need to receive treatment.

Case 2. Take $(\alpha, \beta, \gamma)=(0.7,0.4,0.3)$. We can obtain the cut relation $\widetilde{R}_{\{(0.7,0.4,0.3)\}}$ (given in Table 4$)$.

Table 4. The cut relation $\widetilde{R}_{\{(0.7,0.4,0.3)\}}$.

\begin{tabular}{cccccccc}
\hline$\widetilde{\boldsymbol{R}}_{\{(\mathbf{0 . 7 , 0 . 4 , 0 . 3 ) \}}}$ & $y_{\mathbf{1}}$ & $y_{\mathbf{2}}$ & $y_{3}$ & $y_{\mathbf{4}}$ & $y_{5}$ & $y_{\mathbf{6}}$ & $y_{\mathbf{7}}$ \\
\hline$x_{1}$ & 0 & 1 & 1 & 0 & 0 & 1 & 0 \\
$x_{2}$ & 1 & 0 & 1 & 0 & 0 & 1 & 0 \\
$x_{3}$ & 0 & 0 & 0 & 0 & 1 & 0 & 1 \\
$x_{4}$ & 1 & 0 & 0 & 0 & 0 & 1 & 0 \\
$x_{5}$ & 0 & 0 & 0 & 0 & 1 & 0 & 1 \\
\hline
\end{tabular}

According to Definition 11, we can get

$\widetilde{R}_{\{(0.7,0.4,0.3)\}}\left(x_{1}\right)=\left\{y_{2}, y_{3}, y_{4}, y_{6}\right\}$,

$\widetilde{R}_{\{(0.7,0.4,0.3)\}}\left(x_{2}\right)=\left\{y_{1}, y_{3}, y_{6}\right\}$,

$\widetilde{R}_{\{(0.7,0.4,0.3)\}}\left(x_{3}\right)=\left\{y_{5}, y_{7}\right\}$,

$\widetilde{R}_{\{(0.7,0.4,0.3)\}}\left(x_{4}\right)=\left\{y_{1}, y_{6}\right\}$,

$\widetilde{R}_{\{(0.7,0.4,0.3)\}}\left(x_{5}\right)=\left\{y_{5}, y_{7}\right\}$.

Then, by Definition 12, we can calculate the lower approximation, the upper approximation, the boundary region and the negative region of $Y$ as follows, respectively.

$$
\begin{aligned}
& \widetilde{R}_{\{(0.7,0.4,0.3)\}}(Y)=\left\{x_{2}, x_{4}\right\}, \\
& \overline{\widetilde{R}}_{\{(0.7,0.4,0.3)\}}(Y)=\left\{x_{1}, x_{2}, x_{4}\right\}, \\
& b n_{\widetilde{R}_{\{(0.7,0.4,0.3)\}}}(Y)=\left\{x_{1}\right\}, \\
& n e g_{\widetilde{R}_{\{(0.7,0.4 .0 .3)\}}}(Y)=\left\{x_{3}, x_{5}\right\} .
\end{aligned}
$$

By Definition 14, we also can calculate the approximate precision of the set $Y(Y \subseteq V)$ as follows:

$\rho_{\widetilde{R}_{\{(\alpha, \beta, \gamma)\}}}(Y)=\frac{2}{3}$.

Thus, we can obtain the following conclusions:

(1) Patients $x_{2}$ and $x_{4}$ are suffering from the disease $Y$ and must receive treatment immediately.

(2) We cannot determine whether patient $x_{1}$ is suffering from the disease $Y$ or not.

(3) Patients $x_{3}$ and $x_{5}$ are not suffering from the disease $Y$ and do not need to receive treatment. 
Based on the above analysis, the proposed model and method could help decision makers make a scientific and precise decision as they face the similar cases in practice. Besides, the model presented in this paper also permits controlling the risk of misdiagnosis in practice.

To explore the effectiveness of the method proposed in this paper, we compare it with the method proposed in [29]. The method given in [29] only deals with the decision making problems with intuitionistic fuzzy information, but not the decision making problems with the single-valued neutrosophic information; while the model proposed in the present paper can handle the decision making problems not only with intuitionistic fuzzy information (since intuitionistic fuzzy sets can be regarded as a special case of SVNSs), but also with single-valued neutrosophic information. Thus, the proposed method is more general, and its application domain is wider than that of the method in [29].

The proposed model is based on the level cut relation of single-valued neutrosophic relations. There are two advantages. One advantage is that the level parameter in the model can control the risk of the misdiagnosis. Another advantage is that the model can approximate the crisp concept by converting a single-valued neutrosophic fuzzy relation into a crisp binary relation.

The rough set method does not depend on any other extra knowledge besides the given dataset. Rough set theory can be applied as a component of hybrid solutions in machine learning and data mining. They have been found to be particularly useful for rule induction and feature selection. Decision makers can control the size of the loss of information by changing the level parameter.

\section{Conclusions}

In the present paper, based on the $(\alpha, \beta, \gamma)$-cut relation $\widetilde{R}_{\{(\alpha, \beta, \gamma)\}}(\alpha, \beta, \gamma \in(0,1])$, we propose a new rough set model in generalized single-valued neutrosophic approximation spaces and obtain two extended models of the model. Some properties are studied. Finally, we use an example to illustrate the proposed rough set model's application in a multi-attribute decision making problem.

Acknowledgments: This work is partially supported by the National Natural Science Foundation of China (Nos. 61473181 and 11526163), the Fundamental Research Funds For the Central Universities (No. GK201702008) and the Youth Academic Innovation Team in Northwest University of Political Science and Law.

Author Contributions: Hai-Long Yang provided the idea of the paper. Yan-Ling Liu proved the theorems. Zhi-Lian Guo analyzed the model's application. Zhi-Lian Guo, Yan-Ling Liu and Hai-Long Yang wrote the paper.

Conflicts of Interest: The authors declare no conflict of interest.

\section{References}

1. Smarandache, F. Neutrosophy. Neutrosophic Probability, Set, and Logic; American Research Press: Rehoboth, NM, USA, 1998.

2. Smarandache, F. A Unifying Field in Logics. Neutrosophy: Neutrosophic Probability, Set and Logic; American Research Press: Rehoboth, NM, USA, 1999.

3. Zadeh, L.A. Fuzzy sets. Inform. Control 1965, 8, 338-353.

4. Turksen, I. Interval valued fuzzy sets based on normal forms. Fuzzy Sets Syst. 1986, 20, 191-210.

5. Atanassov, K. Intuitionistic fuzzy sets. Fuzzy Sets Syst. 1986, 20, 87-96.

6. Atanassov, K.; Gargov, G. Interval valued intuitionistic fuzzy sets. Fuzzy Sets Syst. 1989, 31, 343-349.

7. Wang, H.; Smarandache, F.; Zhang, Y.Q.; Sunderraman, R. Single valued neutrosophic sets. Multispace Multistruct. 2010, 4, 410-413.

8. Ye, J. Multicriteria decision making method using the correlation coefficient under single-valued neutrosophic enviroment. Int. J. Gen. Syst. 2013, 42, 386-394.

9. Ye, J. Improved correlation coefficients of single valued neutrosophic sets and interval neutrosophic sets for multiple attributedecision making. J. Intell. Fuzzy Syst. 2014, 27, 2453-2462.

10. Baušys, R.; Zavadskas, E.K.; Kaklauskas, A. Application of neutrosophic set to multi-criteria decision making by copras. J. Econ. Comput. Econ. Cybern. Stud. Res. 2015, 49, 91-105. 
11. Zavadskas, E.K.; Baušys, R.; Lazauskas, M. Sustainable assessment of alternative sites for the construction of a waste incineration plant by applying WASPAS method with single-valued neutrosophic set. Sustainability 2015, 7, 15923-15936.

12. Zavadskas, E.K.; Baušys, R.; Stanujkic, D.; Magdalinovic-Kalinovic, M. Selection of lead-zinc flotation circuit design by applying WASPAS method with single-valued neutrosophic set. Acta Montan. Slovaca 2016, 21, 85-92.

13. Zavadskas, E.K.; Baušys, R.; Kaklauskas, A.; Ubartè, I.; Kuzminskè, A.; Gudienè, N. Sustainable market valuation of buildings by the single-valued neutrosophic MAMVA method. Appl. Soft Comput. 2017, $57,74-87$.

14. Li, Y.H.; Liu, P.D.; Chen, Y.B. Some single-valued neutrosophic number heronian mean operators and their application in multiple attribute group decision making. Informatica 2016, 27, 85-110.

15. Baušys, R.; Juodagalvienė, B. Garage location selection for residential house by WASPAS-SVNS method. J. Civ. Eng. Manag. 2017, 23, 421-429.

16. Ye, J. Similarity measures between interval neutrosophic sets and their applications in multi-criteria decision making. J. Intell. Fuzzy Syst. 2014, 26, 165-172.

17. Ye, J. Vector Similarity measures of simplified neutrosophic sets and their application in multi-criteria decision making. Int. J. Fuzzy Syst. 2014, 16, 204-211.

18. Majudar, P.; Samanta, S.K. On similarity and entropy of neutrosophic sets. J. Intell. Fuzzy Syst. 2014, 26, 1245-1252.

19. Peng, J.J.; Wang, J.Q.; Zhang, H.Y.; Chen, X.H. An outranking approach for multi-criteria decision making problems with simplified neutrosophic sets. Appl. Soft Comput. 2014, 25, 336-346.

20. Liu, P.D.; Wang, Y.M. Interval neutrosophic prioritized OWA operator and its application to multiple attribute decision making. J. Syst. Sci. Complex. 2016, 29, 681-697.

21. Zhang, C.; Zhai, Y.; Li, D.; Mu, Y. Steam turbine fault diagnosis based on single-valued neutrosophic multi-granulation rough sets over two universes. J. Intell. Fuzzy Syst. 2016, 30, 2829-2837.

22. Sahin, R. Cross-entropy measure on interval neutrosophic sets and its applications in multi-criteria decision making. Neural Comput. Appl. 2017, 8, 1177-1187.

23. Ye, J.; Fu, J. Multi-period medical diagnosis method using a single-valued neutrosophic similarity measure based on tangent function. Comput. Methods Progr. Biomed. 2016, 123, 142-149.

24. Yang, H.L.; Guo, Z.L.; She, Y.H.; Liao, X.W. On single-valued neutrosophic relations. J. Intell. Fuzzy Syst. 2016, 30, 1045-1056.

25. Ye, J. Simplified neutrosophic harmonic averaging projection-based method for multiple attribute decision making problems. Int. J. Mach. Learn. Cybern. 2017, 8, 981-987.

26. Stanujkic, D.; Zavadskas, E.K.; Smarandache F.; Brauers W.K.M.; Karabasevic D. A neutrosophic extension of the MULTIMOORA method. Informatica 2017, 28, 181-192.

27. Pawlak, Z. Rough sets. Int. J. Comput. Inform. Sci. 1982, 11, 341-356.

28. Pawlak, Z. Rough Sets: Theoretical Aspects of Reasoning about Data; Kluwer Academic Publishers: Dordrecht, The Netherlands; Boston, MA, USA, 1991.

29. Guo, Z.L.; Yang, H.L.; Wang, J. Rough set over dual-universes in intuitionistic fuzzy approximation space and its application. J. Intell. Fuzzy Syst. 2015, 28, 169-178.

30. Li, T.J.; Zhang, W.X. Rough fuzzy approximation on two universes of discourse. Inform. Sci. 2008, 178, 892-906.

31. Mareay, R. Rough and rough fuzzy sets on two universes via covering approach. J. Intell. Fuzzy Syst. 2016, 30, 1139-1146.

32. Shen, Y.; Wang, F. Variable precision rough set model over two universes and its properties. Soft Comput. 2011, 15, 557-567.

33. Sun, B.Z.; Ma, W.M.; Liu, Q. An approach to decision making based on intuitionistic fuzzy rough sets over two universes. J. Oper. Res. Soc. 2013, 64, 1079-1089.

34. Yan, R.; Zheng, J.; Liu, J.; Zhai, Y. Research on the model of rough set over dual-universes. Knowl. Based Syst. 2010, 23, 817-822.

35. Yan, R.; Zheng, J.; Liu, J.; Qin, C. Rough set over dual-universes in fuzzy approximation space. Iran. J. Fuzzy Syst. 2012, 9, 79-91. 
36. Yang, H.L.; Li, S.G.; Wang, S.Y.; Wang, J. Bipolar fuzzy rough set model on two different universes and its application. Knowl. Based Syst. 2012, 35, 94-101.

37. Yang, H.L.; Li, S.G.; Guo, Z.L.; Ma, C.H. Transformation of bipolar fuzzy rough set models. Knowl. Based Syst. 2012, 27, 60-68.

38. Yang, H.L.; Liao, X.W.; Wang, S.Y.; Wang, J. Fuzzy probabilistic rough set model on two universes and its applications. Int. J. Approx. Reason. 2013, 54, 1410-1420.

39. Yao, Y.Y.; Wong, S.K.M.; Wang, L.S. A non-numeric approach to uncertain reasoning. Int. J. Gen. Syst. 1995, 23, 343-359.

40. Salama, A.A.; Broumi, S. Roughness of neutrosophic sets. Elix. Appl. Math. 2014, 74, 26833-26837.

41. Broumi, S.; Smarandache, F.; Dhar, M. Rough neutrosophic sets. Neutrosophic Sets Syst. 2014, 3, $62-67$.

42. Broumi, S.; Smarandache, F. Single Valued Neutrosophic Information Systems Based on Rough Set Theory. Available online: https:/ /www.researchgate.net/publication/280742564 (accessed on 7 August 2015).

43. Broumi, S.; Smarandache, F. Interval neutrosophic rough set. Neutrosophic Sets Syst. 2015, 7, $23-31$.

44. Yang, H.L.; Zhang, C.L.; Guo, Z.L.; Yiu, Y.L.; Liao, X.W. A hybrid model of single-valued neutrosophic sets and rough sets: Single valued neutrosophic rough set model. Soft Comput. 2016, doi:10.1007/s00500016-2356-y.

45. Ye, J. A multi-criteria decsion-making method using aggregation operators for simplified neutrosophic sets. J. Intell. Fuzzy Syst. 2014, 26, 2459-2466.

(c) 2017 by the authors. Licensee MDPI, Basel, Switzerland. This article is an open access article distributed under the terms and conditions of the Creative Commons Attribution (CC BY) license (http:/ / creativecommons.org/licenses/by/4.0/). 